University of Nebraska - Lincoln

DigitalCommons@University of Nebraska - Lincoln

\title{
Three-dimensional Bayesian geostatistical aquifer characterization at the Hanford 300 Area using tracer test data
}

\author{
Xingyuan Chen \\ Pacific Northwest National Laboratory, xingyuan.chen@pnnl.gov \\ Haruko Murakami \\ University of California - Berkeley \\ Melanie S. Hahn \\ University of California - Berkeley \\ Glenn E. Hammond \\ Pacific Northwest National Laboratory \\ Mark Rockhold \\ Pacific Northwest National Laboratory \\ See next page for additional authors
}

Follow this and additional works at: https://digitalcommons.unl.edu/usdoepub

Part of the Bioresource and Agricultural Engineering Commons

Chen, Xingyuan; Murakami, Haruko; Hahn, Melanie S.; Hammond, Glenn E.; Rockhold, Mark; Zachara, John M.; and Rubin, Yoram, "Three-dimensional Bayesian geostatistical aquifer characterization at the Hanford 300 Area using tracer test data" (2012). US Department of Energy Publications. 309.

https://digitalcommons.unl.edu/usdoepub/309

This Article is brought to you for free and open access by the U.S. Department of Energy at DigitalCommons@University of Nebraska - Lincoln. It has been accepted for inclusion in US Department of Energy Publications by an authorized administrator of DigitalCommons@University of Nebraska - Lincoln. 


\section{Authors}

Xingyuan Chen, Haruko Murakami, Melanie S. Hahn, Glenn E. Hammond, Mark Rockhold, John M. Zachara, and Yoram Rubin 


\title{
Three-dimensional Bayesian geostatistical aquifer characterization at the Hanford 300 Area using tracer test data
}

\author{
Xingyuan Chen, ${ }^{1,2}$ Haruko Murakami, ${ }^{3}$ Melanie S. Hahn, ${ }^{1}$ Glenn E. Hammond, ${ }^{2}$ \\ Mark L. Rockhold, ${ }^{2}$ John M. Zachara, ${ }^{2}$ and Yoram Rubin ${ }^{1}$ \\ Received 15 March 2011; revised 13 February 2012; accepted 18 April 2012; published 1 June 2012.
}

[1] Tracer tests performed under natural or forced gradient flow conditions can provide useful information for characterizing subsurface properties, through monitoring, modeling, and interpretation of the tracer plume migration in an aquifer. Nonreactive tracer experiments were conducted at the Hanford 300 Area, along with constant-rate injection tests and electromagnetic borehole flowmeter tests. A Bayesian data assimilation technique, the method of anchored distributions (MAD) (Rubin et al., 2010), was applied to assimilate the experimental tracer test data with the other types of data and to infer the threedimensional heterogeneous structure of the hydraulic conductivity in the saturated zone of the Hanford formation.In this study, the Bayesian prior information on the underlying random hydraulic conductivity field was obtained from previous field characterization efforts using constant-rate injection and borehole flowmeter test data. The posterior distribution of the conductivity field was obtained by further conditioning the field on the temporal moments of tracer breakthrough curves at various observation wells. MAD was implemented with the massively parallel three-dimensional flow and transport code PFLOTRAN to cope with the highly transient flow boundary conditions at the site and to meet the computational demands of MAD. A synthetic study proved that the proposed method could effectively invert tracer test data to capture the essential spatial heterogeneity of the three-dimensional hydraulic conductivity field. Application of MAD to actual field tracer data at the Hanford 300 Area demonstrates that inverting for spatial heterogeneity of hydraulic conductivity under transient flow conditions is challenging and more work is needed.

Citation: Chen, X., H. Murakami, M. S. Hahn, G. E. Hammond, M. L. Rockhold, J. M. Zachara, and Y. Rubin (2012), Threedimensional Bayesian geostatistical aquifer characterization at the Hanford 300 Area using tracer test data, Water Resour. Res., 48, W06501, doi:10.1029/2011WR010675.

\section{Introduction}

[2] Spatial variability of subsurface hydrogeological properties, such as hydraulic conductivity, plays a critical role in groundwater flow and transport modeling [Rubin, 2003; Sudicky et al., 2010]. An accurate map of such properties forms the basis for understanding more complex physical, chemical, and microbiological processes [e.g., Scheibe et al., 2001]. The prohibitive cost of collecting sufficient direct point-scale measurements (e.g., core samples) to characterize heterogeneity has motivated extensive research in groundwater inverse modeling (see reviews by McLaughlin and Townley [1996] and Vrugt et al. [2008]),

\footnotetext{
${ }^{1}$ Department of Civil and Environmental Engineering, University of California, Berkeley, California, USA.

${ }^{2}$ Pacific Northwest National Laboratory, Richland, Washington, USA.

${ }^{3}$ Department of Nuclear Engineering, University of California, Berkeley, California, USA.

Corresponding author: X. Chen, Now at Pacific Northwest National Laboratory, PO Box 999, Richland, WA 99352, USA. (xingyuan.chen@pnnl.gov)

C2012. American Geophysical Union. All Rights Reserved. 0043-1397/12/2011WR010675
}

which utilizes indirect data that can be obtained more easily, such as pumping tests and tracer tests, for inferring the hydrogeologic parameters. Geostatistical inverse techniques (see reviews by Zimmerman et al. [1998] and Hendricks Franssen et al. [2009]), which aim at inferring the spatial distribution of hydrogeologic properties using indirect data, have been particularly useful for improving the characterization of spatially variable hydrogeologic properties.

[3] Among the difficulties shared by all geostatistical inversion techniques, the two most challenging ones include the heavy computational burden associated with data inversion and the problem of assimilating multiple types of available data [Medina and Carrera, 2003]. The first difficulty stems from the large number of parameters in geostatistical inverse problems, whereas the latter stems from the absence of a framework that can assimilate multiple types of data while recognizing the multiple scales of measurements they represent. The method of anchored distribution (MAD) [Rubin et al., 2010] was developed to deal with the latter challenge. MAD is a general Bayesian inverse modeling technique that can systematically assimilate multiple sources and scales of data into aquifer characterization. Its modular 
structure enables it to handle complex relationships between data and target variables.

[4] In MAD, the vector of target variables (or parameters) includes structural parameters (such as mean and variogram parameters) that describe the spatial pattern of the heterogeneous property and probability distributions of the target property at selected locations, referred to as anchors. The anchors are used to capture local heterogeneity and serve as conditioning points when we generate random fields over the model domain. Unlike most of the other geostatistical inversion methods, such as those reviewed by Hendricks Franssen et al. [2009] and Bayesian geostatistical inversion based on kriging or cokriging [e.g., Kitanidis, 1995; Shlomi and Michalak, 2007; Fienen et al., 2008], MAD yields a posterior joint distribution of the parameters rather than identifying a single optimal set of parameters. The posterior distribution of parameters, which is nonparametric and is not subject to model linearization or Gaussian confidence interval assumption, not only accounts for the nonuniqueness of the parameters of interest [cf. McKenna et al., 2003; de Barros et al., 2009; Nowak et al., 2010], but also enables accurate quantification of uncertainty. MAD shares the challenge of computational burden with other geostatistical inversion methods. Nevertheless, recent developments in high-performance computing have made the computational cost more affordable. For groundwater flow and transport problems, the parallel three-dimensional reactive flow and transport code PFLOTRAN [Hammond and Lichtner, 2010] can be used to alleviate the computational burden through parallel domain decomposition on supercomputing resources. Its multirealization simulation capability is especially well-suited for MAD implementation, since MAD requires forward simulations on a large number of realizations of random fields.

[5] In this paper, we employ MAD for characterization of the hydraulic conductivity field at the Integrated Field Research Challenge (IFRC) site in U.S. Department of Energy's Hanford 300 Area (available at http://ifchanford. pnl.gov). The Hanford IFRC project is an interdisciplinary project investigating multiscale reactive transport and mass transfer processes associated with a uranium plume, the persistence of which has been attributed to a continuous uranium source in the lower vadose zone and to a combination of complex physical and geochemical processes [Peterson et al., 2008]. In order to evaluate scientific hypotheses regarding uranium geochemistry and rate-limited mass transfer processes, it is critical to characterize the threedimensional (3-D) hydraulic conductivity field. MAD is an ideal tool for integrating various data at the site to estimate the underlying heterogeneous hydraulic conductivity field. Murakami et al. [2010] implemented MAD to estimate the 3 -D hydraulic conductivity field conditioned on constantrate injection tests and electromagnetic borehole flowmeter (referred to as flowmeter hereafter) tests. This study extends the work of Murakami et al. [2010] by expanding the database to include data from the tracer experiment conducted at the site in March 2009.

[6] Tracer test data have been shown to provide valuable information on the spatial distribution of the hydraulic conductivity [Harvey and Gorelick, 1995a; Woodbury and Rubin, 2000; Hendricks Franssen et al., 2003; Nowak and Cirpka, 2006; Fu and Gómez-Hernández, 2009;
Fienen et al., 2009]. However, among the various types of data that are only indirectly related to the hydraulic conductivity, including pressure head and geophysical data, the field concentration data are used less often for geostatistical aquifer characterization. There are several reasons for this underutilization [c.f. Ezzedine and Rubin, 1996; Wilson and Rubin, 2002; Bellin and Rubin, 2004]. One reason is the difficulty in choosing a measurement procedure that best suits the site (e.g., volume-averaging versus fluxaveraging, bailing versus pumping), which makes it difficult to interpret concentration measurements and to model them (we will provide an example later in this paper). Another reason is the discrepancy between the small spatial scale of the measurement device and the support volume they represent, on one hand, and the scale of the numerical grid block, on the other [cf. Rubin et al., 1999, 2003; de Barros and Rubin, 2011]. This discrepancy is usually ignored in applications (see discussion by Ezzedine and Rubin [1996]), because addressing it would require a large number of small grid blocks, with dimensions on the order of the concentration's measurement device, which would lead in turn to heavy computational burdens. With very few examples of 3-D field or laboratory sandbox applications [e.g., Lavenue and de Marsily, 2001; Kollat et al., 2008, 2011], inverse modeling thus far has mainly been conducted in two dimensions, usually in the horizontal plane [e.g., Rubin and Dagan, 1987; Harvey and Gorelick, 1995a, 1995b; Hendricks Franssen et al., 2003; Nowak and Cirpka, 2006; Fu and Gómez-Hernández, 2009]. Such applications rely likewise on 2-D data such as transmissivity and vertically averaged pressure heads. Although 2-D application is appealing because of its relatively light computational burden and can be justified under restrictive conditions, concentration data in many cases are measured over limited vertical intervals that would require modeling in three dimensions at a high computational cost. Hence, including concentrations in the database for inversion requires a 3-D formulation, which would increase the computational burden by orders of magnitude. This requires that special consideration be given to the computational aspects. Recent developments in Monte Carlo-based geostatistical inverse modeling techniques, including MAD, and advances in computing power have enabled the implementation of more sophisticated and computationally intensive conceptual models within inverse modeling frameworks.

[7] The objectives of this study are to apply MAD to condition the aquifer characterization on tracer test data as well as on two types of hydraulic tests (constant-rate injection tests and flowmeter tests) and assess improvement by comparing inversion results with and without the tracer data, to address the challenges of implementing the proposed framework on real field data due to the complex field conditions, and to show the potential of using highperformance computing to tackle the computational burden stemming from the nature of Monte Carlo-based geostatistical inversion techniques (including MAD) or the complexity of the conceptual model. In our work, full 3-D simulation of flow and transport processes are necessary due to the extremely dynamic flow conditions caused by the river stage fluctuations in the adjacent Columbia River. Considerable uncertainty exists in conceptualizing this boundary condition, and as a result, we first verify the proposed framework 
using a synthetic study that mimics the field conditions, and then present the results of assimilating the real experimental data. We focus on studying the parameter uncertainty, i.e., no conceptual model uncertainty is considered in the present study.

\section{Site Conditions and Experiment Description}

[8] The Hanford 300 Area Integrated Field Research Challenge (IFRC) site is located in southeastern Washington State. The site is within the footprint of a former disposal facility for uranium-bearing liquid wastes known as the South Process Pond, $\sim 250 \mathrm{~m}$ west of the Columbia River. The groundwater table at the site is highly variable in response to river stage fluctuations, ranging 2-3 $\mathrm{m}$ or more annually and averaging $0.5 \mathrm{~m}$ diurnally.

[9] The main lithology at the site is a poorly sorted mixture of sediments dominated by gravel up to boulder size [Bjornstad et al., 2009]. This highly permeable and coarsegrained Hanford formation is underlain by the Ringold formation, whose upper portion is a discontinuous low-permeability layer consisting of cohesive and compacted fine sand to silty sand. An example cross section in the vicinity of the IFRC well field is provided in Figure 1 to show these stratigraphic units. The portion marked as "South Process Pond" is most relevant to this study. Our focus is on the saturated portion of the Hanford formation, the thickness of which is variable over the site due to groundwater table fluctuations, ranging from $\sim 5 \mathrm{~m}$ to $\sim 8 \mathrm{~m}$.

[10] The porosities of the Hanford and Ringold formation sediments have been estimated from a limited number of intact core samples from within the IFRC well field, and from a larger number of intact core samples obtained in previous drilling and sampling efforts at the 300 Area [Williams et al. 2008]. We assumed that the average total porosity value is 0.2 in the saturated portion of the Hanford formation, as recommended by Williams et al. [2008]. We are aware that the heterogeneity of porosity can affect the estimated hydraulic conductivity field. However, there is currently not enough information for us to define a spatially variable porosity field. Compared to the variability in hydraulic conductivity (orders of magnitude difference), the variability in porosity should have a secondary effect on transport. The error introduced by not explicitly modeling the spatial variability of porosity is incorporated into the uncertainty in the estimated anchors and structural parameters describing the variability in hydraulic conductivity. Ongoing geophysical characterization at the site may contribute to the development of a heterogeneous porosity field that can be incorporated in a future study.

[11] The experimental well field at the IFRC site is depicted in Figure 2. The triangular design was due to the highly variable groundwater flow direction. Well 2-9 is chosen as the primary injection well as the dominant flow direction is southeast and parallel to the axis of the well field passing through wells 2-10 and 3-29. Most of the wells are $\sim 20 \mathrm{~m}$ deep and are screened over the entire saturated portion of the Hanford formation. There are also three multilevel well clusters screened over three different depth intervals to provide depth-discrete monitoring. The shallow wells are screened over a $1.53-\mathrm{m}(5-\mathrm{ft})$ interval located at $\sim 9.14-10.67 \mathrm{~m}$ below ground surface, the intermediate wells are screened over a $0.61-\mathrm{m}(2-\mathrm{ft})$ interval located at $\sim 12.86-13.47 \mathrm{~m}$ below ground surface, and the deep wells are screened over a $0.61-\mathrm{m}$ (2-ft) interval located at $\sim 16.46-17.07 \mathrm{~m}$ below ground surface.

[12] One complication at the site is the vertical wellbore flow in fully screened wells induced by the river stage fluctuations in the Columbia River, which was later observed at the site during the long-term monitoring [Newcomer et al., 2010]. The vertical wellbore flow, as illustrated in Figure 3 and discussed by Zachara [2010] and Vermuel et al. [2010], occurs within long-screened wells in layered media where the wells act as conduits for intercommunication between shallow and deep, high-permeability zones that are separated by a zone of lower permeability. This study demonstrated that when contaminant concentrations within the aquifer vary significantly over the depth interval interrogated, river-induced vertical wellbore flow can result in variations in measured concentration that nearly encompass the full range of variation in aquifer contaminant distribution with depth. The difficulty in the assimilation of tracer data caused by this complication will be discussed later.

[13] As a part of the hydrologic characterization of the conductivity field, 14 constant-rate injection tests were conducted in fully screened wells. Each test had one injection well and seven to 10 observation wells. There were reliable flowmeter tests data available from 19 (out of 26 tested) fully screened wells, which yielded 283 depth-discrete relative hydraulic conductivities with depth intervals of 0.3-0.6 m [Murakami et al., 2010]. The interpretation of

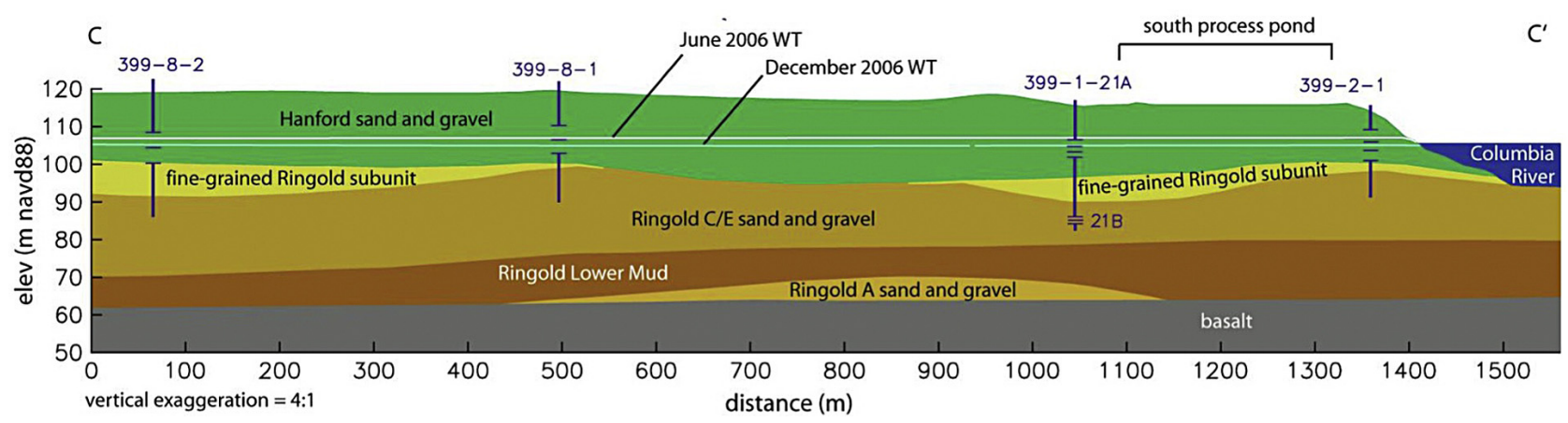

Figure 1. Cross section through the 300 Area showing major hydrostratigraphic units [from Williams et al., 2008]. WT denotes the water table. Ringold C/E denotes Ringold unit C or E. 


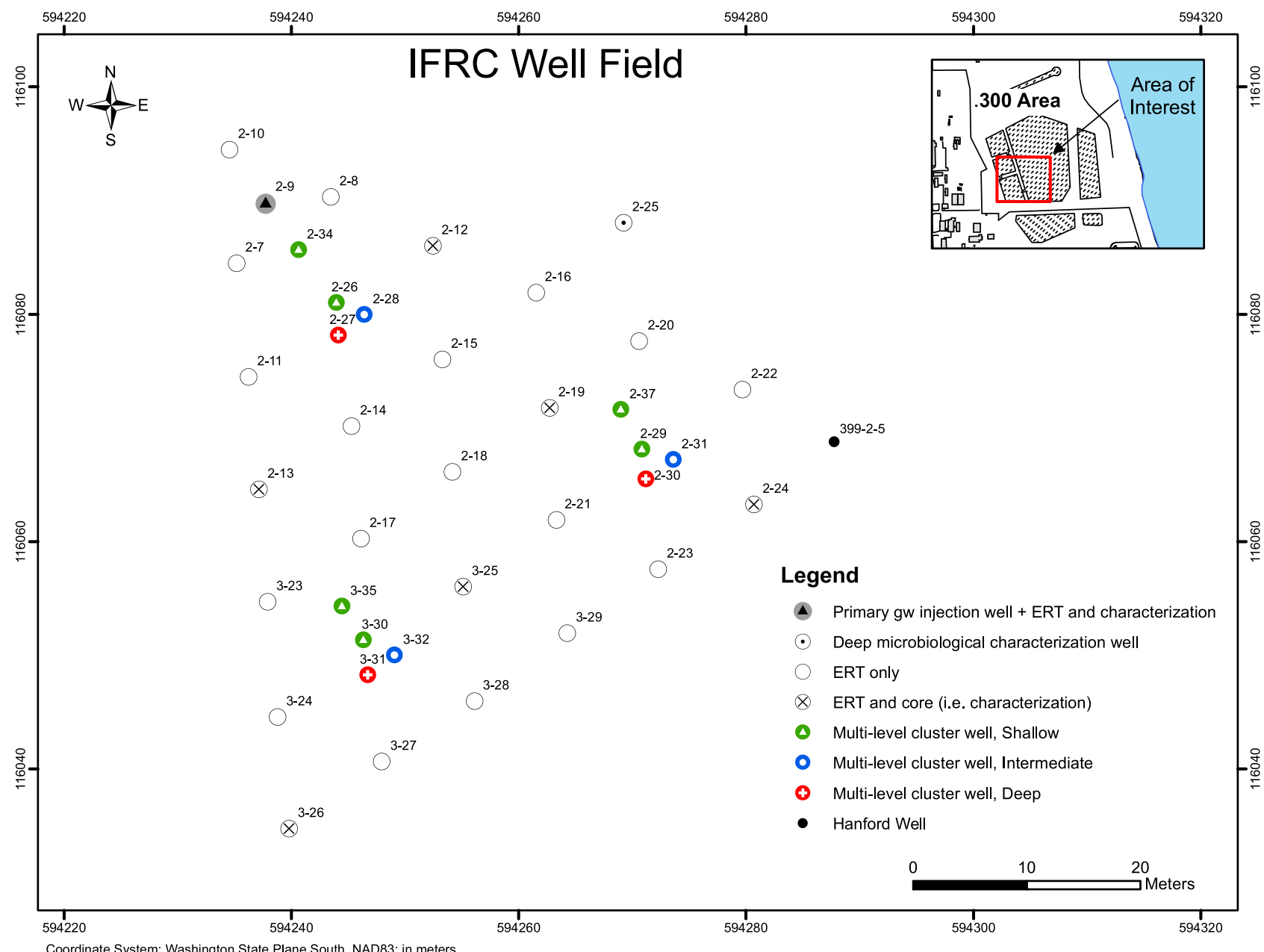

Figure 2. IFRC well field (provided by Rob Mackley, Environmental Systems Group, Pacific Northwest National Laboratory).

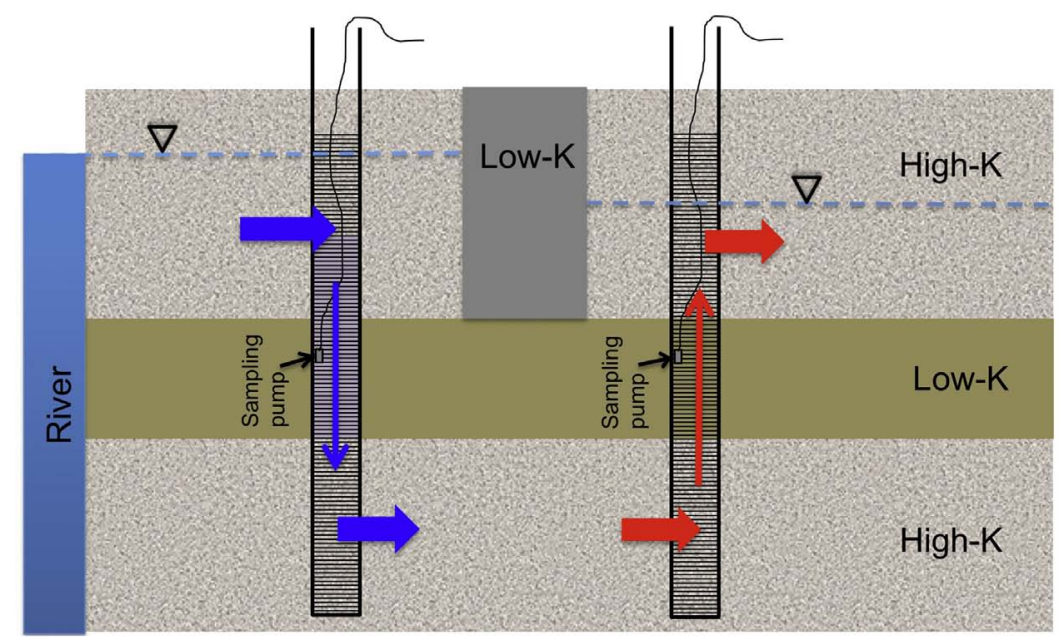

Figure 3. Illustration of downward and upward wellbore flow when river water rises. A flow barrier, which could be relatively more permeable than the low-K zone in the layered material, within the upper zone would impose pressure gradients on wells penetrating the low-K zone. Water flows preferentially through the well to bypass the low-K zone. When river stage rises, water would flow downward within the boreholes on the river side of the barrier, and upward on the opposite side. The flow direction flips when the river stage falls. 
the flowmeter data using the standard procedure suggested by Molz et al. [1994] could be affected by the dynamics of the vertical wellbore flow, which may not be fully accounted for by subtracting an ambient flowmeter profile from the dynamic profile obtained during pumping, since ambient flow conditions could be changing during the dynamic test. The results from wells that experienced significant change in ambient flow during the flowmeter test were excluded in the study of Murakami et al. [2010] and in this study as well. The vertical profiles from the flowmeter tests indicate a less permeable layer over the central third of the Hanford formation at many of the wells, with variable thickness and contact depths across the site. More detailed descriptions of the site and hydrologic tests are provided by Bjornstad et al. [2009].

[14] Two field-scale nonreactive tracer tests were performed at the site in November 2008 and March 2009, respectively. The first test took place during a period when the adjacent Columbia River experienced large water level fluctuations. The second test was started on 13 March 2009 , during which the river stage was relatively stable. We focus on the second test (referred to as the March 09 tracer test hereinafter) in this study.

[15] In the March 09 tracer test, a bromide solution $\left(\mathrm{Br}^{-}\right)$ with an average concentration of $95 \mathrm{mg} \mathrm{L}^{-1}$ was injected into wells $2-9$ for $9.5 \mathrm{~h}$ at a nearly constant rate of $4.52 \times$ $10^{-3} \mathrm{~m}^{3} \mathrm{~s}^{-1}$. The total injected volume was $153.7 \mathrm{~m}^{3}$. After the injection stopped, the plume was allowed to drift under natural gradient conditions, and it was tracked for several weeks. The tracer plume was monitored by collecting aqueous samples at selected monitoring wells over time, and the samples were analyzed to quantify $\mathrm{Br}^{-}$concentration.

\section{Methodology}

[16] To incorporate the tracer-test data into the characterization efforts of the heterogeneous hydraulic conductivity field, we used MAD, which is designed to assimilate multitype multiscale data sets that are related directly or indirectly to the target variables (e.g., conductivity, porosity). MAD also does not require assumptions (e.g., Gaussianity) about the statistical distributions of parameters or likelihood function. It is thus suitable for integrating multiple and complementary types of data into aquifer characterization. A major advantage of the MAD framework is that it can be implemented sequentially to assimilate data that are generated at different times, without having to discard assimilation results that are completed prior to the availability of new data. We refer readers to Rubin et al. [2010] for details of MAD, while its underlying principles are summarized here for the sake of completeness.

\subsection{MAD Framework}

[17] In the MAD framework, we denote the spatial property of interest by $\mathbf{Y}(\mathbf{x})$, which is a space random function that describes the spatial variability as a point process, with $\mathbf{x}$ being the space coordinate. We further denote a realization of the entire field of $\mathbf{Y}$ by $\tilde{\mathbf{Y}}$, and it is defined through a vector of parameters $\{\boldsymbol{\theta}, \boldsymbol{\theta}\}$, where $\boldsymbol{\theta}$ represents geostatistical structural parameters that capture the global features of $\tilde{\mathbf{Y}}$ (e.g., mean and correlation structure of the field) and $\boldsymbol{\vartheta}$ denotes anchored distributions or anchors in short. The anchors are given in the form of statistical distributions of the property at known or chosen locations, and they are used as devices for capturing local heterogeneity of $\tilde{\mathbf{Y}}$ that cannot be modeled by the structural parameters.

[18] MAD employs a systematic classification of data available in a specific study as a major tool for data assimilation. Data that gives point values of $\mathbf{Y}$ directly or via empirical relations are classified as type A, and data that are related to $\mathbf{Y}$ indirectly through a large-sale physical process are classified as type B. If our variable of interest is hydraulic conductivity, then examples of type-A data could be permeameter test data or grain size distributions, and examples of type-B data could be drawdown curves recorded during a pumping test or tracer concentrations measured during a tracer test. We denote all available data by $\mathbf{z}$ : type-A data by $\mathbf{z}_{a}$, and type-B data by $\mathbf{z}_{b}$.

[19] MAD defines two types of anchors corresponding to its data classification. Type-A anchors $\left(\boldsymbol{\vartheta}_{a}\right)$, which are placed at the same locations as the type-A data, are given as exact values assuming no measurement error or in the form of statistical distributions about the local measurements to account for measurement (or regressional) errors. Type-B anchors $\left(\boldsymbol{\vartheta}_{b}\right)$, which are placed at selected locations, are used to capture information from type-B data. Since type-B data, such as drawdown curves obtained from pumping tests, are influenced by the spatial property over an area, multiple type-B anchors can be placed for each type-B measurement based on sensitivity analysis, geological conditions, or the locations that are beneficial to prediction (details provided by Rubin et al. [2010]). We denote the entire anchor set by $\boldsymbol{\vartheta}=\left\{\boldsymbol{\vartheta}_{a}, \boldsymbol{\vartheta}_{b}\right\}$.

[20] In the MAD framework, the goal of the inversion is to determine a posterior distribution of the model parameters conditioned on the data, i.e., $p(\boldsymbol{\theta}, \boldsymbol{\theta} \mid \mathbf{z})$. Once this distribution is determined, any random draw of $\{\boldsymbol{\theta}, \boldsymbol{\theta}\}$ from this distribution contains all of the information needed for generating a random realization of $\tilde{\mathbf{Y}}$.

[21] Using Bayes' rule, the posterior distribution can be derived as,

$$
\begin{aligned}
& p\left(\boldsymbol{\theta}, \boldsymbol{\vartheta} \mid \mathbf{z}_{a}, \mathbf{z}_{b}\right) \propto p\left(\mathbf{z}_{b} \mid \boldsymbol{\theta}, \boldsymbol{\vartheta}, \mathbf{z}_{a}\right) p\left(\boldsymbol{\theta}, \boldsymbol{\vartheta} \mid \mathbf{z}_{a}\right), \\
& =p\left(\mathbf{z}_{b} \mid \boldsymbol{\theta}, \boldsymbol{\vartheta}\right) p\left(\boldsymbol{\vartheta}_{b} \mid \boldsymbol{\theta}, \boldsymbol{\vartheta}_{a}\right) p\left(\boldsymbol{\theta} \mid \boldsymbol{\vartheta}_{a}\right) p\left(\boldsymbol{\vartheta}_{a} \mid \mathbf{z}_{a}\right)
\end{aligned}
$$

where $p\left(\mathbf{z}_{b} \mid \boldsymbol{\theta}, \boldsymbol{9}\right)$ is the likelihood of type-B data, the distribution $p\left(\boldsymbol{\vartheta}_{a} \mid \mathbf{z}_{a}\right)$ is the distribution of type-A anchors conditioned on type-A data, the distribution $p\left(\boldsymbol{\theta} \mid \boldsymbol{\vartheta}_{a}\right)$ can be determined using model-based geostatistical approach [Diggle and Ribeiro, 2006], and the distribution $p\left(\boldsymbol{\vartheta}_{b} \mid \boldsymbol{\theta}, \boldsymbol{\vartheta}_{a}\right)$ is the prior of type- $\mathrm{B}$ anchors given type-A data and structural parameters.

[22] The likelihood function in equation (1) is the key to relating the posterior distribution of the model parameters with the type-B data. It can be obtained in one of two ways. It can either be assumed or derived from physical principles using statistical modeling assumptions [Hoeksema and Kitanidis, 1984; Dagan, 1985; Rubin and Dagan, 1987], or it can be determined nonparametrically by generating an ensemble of $\mathbf{z}_{b}$ for any given $\{\boldsymbol{\theta}, \boldsymbol{\vartheta}\}$. Both approaches can be implemented in MAD. The flexibility of using a nonparametric likelihood estimation differs MAD fundamentally from other Bayesian methods, such as the ensemble Kalman filter (EnKF) [Evensen, 1994, 2003] and generalized likelihood 
uncertainty estimation (GLUE) [Beven and Binley, 1992; Beven, 2007; Mantovan and Todini, 2006], both of which require a functional form of likelihood. However, MAD is more computationally intensive than EnKF, GLUE, as well as other non-Bayesian geostatistical inversion techniques, such as pilot points [RamaRao et al., 1995; Lavenue et al., 1995; Alcolea et al., 2006] and the sequential self-calibration method [Sahuquillo et al., 1992; Gómez-Hernández et al., 1997; Capilla et al., 1997]. A detailed comparison on the similarities and differences between MAD and pilot points method has been discussed by Rubin et al. [2010].

[23] We show in Figure 4 a flowchart of MAD adapted from Rubin et al. [2010], in which we summarize the three main components of implementing MAD in any inverse problem: a prior distribution of parameters conditioned on type-A data (the preprocessing block), a forward simulator (the forward simulation block), and a likelihood estimation. The derived posterior distribution of parameters can be used to generate random fields for predictions (the prediction block). Another necessary step is to place type-B anchors at locations where the local heterogeneity is important and is sensitive to the type- $\mathrm{B}$ data (details provided by Rubin et al. [2010]). It is clear in this flowchart that type-A data is used to estimate the prior distribution of parameters, whereas the type-B data is integrated in the likelihood estimation. We address the implementation details of these elements in detail in the following subsections.

\subsection{Implementation of MAD}

\subsubsection{Prior Distribution of Parameters and Anchor Placement}

[24] Prior to assimilating the tracer experimental data, we first assimilated the constant-rate injection tests and flowmeter tests in a previous study [Murakami et al., 2010], which provides the prior distribution of the parameters for assimilating the tracer test data. As studied earlier by Li et al. [2008], combining the flowmeter profiles (that provide relative vertical distributions of hydraulic conductivity) and pumping tests (or injection tests in our study, that contain information about the depth-averaged hydraulic conductivity) enables 3-D geostatistical inversion of otherwise 2-D injection tests. In summary, the joint analysis of the injection tests and flowmeter tests provided us with a joint distribution of 3-D structural parameters $(\boldsymbol{\theta})$, including mean $(\beta)$, variance $\left(\eta^{2}\right)$, horizontal scale $\left(\lambda_{h}\right)$, vertical scale $\left(\lambda_{v}\right)$, and nugget $\left(v^{2}\right)$ of the field, depth-discrete anchors at the flowmeter data locations, adopted as type-A anchors with measurement errors $\left(\boldsymbol{\vartheta}_{a}\right)$, and depth-average conductivity values at the wells without flowmeter data $\left(\mathbf{K}_{A}\right)$. This distribution is denoted by $p\left(\boldsymbol{\theta}, \boldsymbol{\vartheta}_{a}, \mathbf{K}_{A} \mid \mathbf{z}_{\mathrm{EBF}}, \mathbf{z}_{\mathrm{INJ}}\right)$, where $\mathbf{z}_{\mathrm{EBF}}$ and $\mathbf{z}_{\mathrm{INJ}}$ are the flowmeter tests data and injection tests data, respectively.

[25] The locations of anchors are depicted in Figure 5, where flowmeter wells contain depth-discrete type-A anchors, and nonflowmeter wells contain depth-average conductivity distributions from assimilating constantinjection tests. The cross-sections of anchor placement along the two transects (T1 and T2) are shown to demonstrate the vertical distribution of anchors. The anchors collocated with flowmeter test data are type-A anchors. We note that there were insufficient flowmeter wells, and thus type-A anchors, along the centerline of the well field (T1), especially in the vicinity of the injection source. Since these regions are important for modeling the tracer plume, we placed additional type-B anchors along the major flow path. At each horizontal location selected for additional type-B anchor placement, there are six anchors placed at different depths to capture the vertical profiles. It can be observed in Figure 5 that flowmeter test data results in a dense distribution of type-A anchors. Although we may have placed as many type-B anchors in additional locations, we started with a relatively sparse vertical distribution to reduce computational cost.

[26] We assume in this study that type-B anchors $\left(\boldsymbol{\vartheta}_{b}\right)$ follow a multivariate normal distribution conditioned on $\boldsymbol{\theta}$ and $\boldsymbol{\vartheta}_{a}$ [Rubin et al., 2010], with conditional mean $\mu_{\boldsymbol{\vartheta}_{b} \mid \boldsymbol{\vartheta}_{a}}$

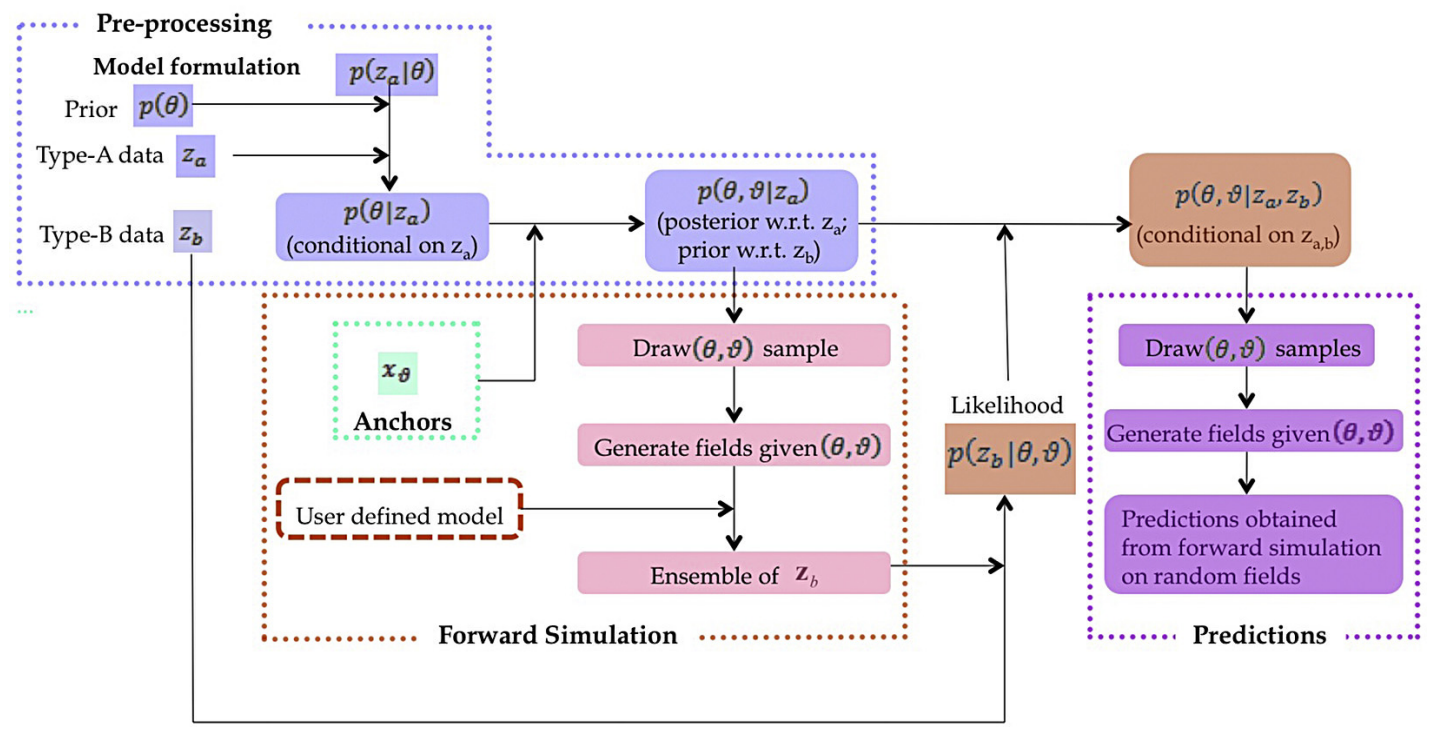

Figure 4. Flowchart of MAD (adapted from Rubin et al. [2010]). 
(a) Plan view



(c) $\mathrm{T} 2$



(b) $\mathrm{T} 1$

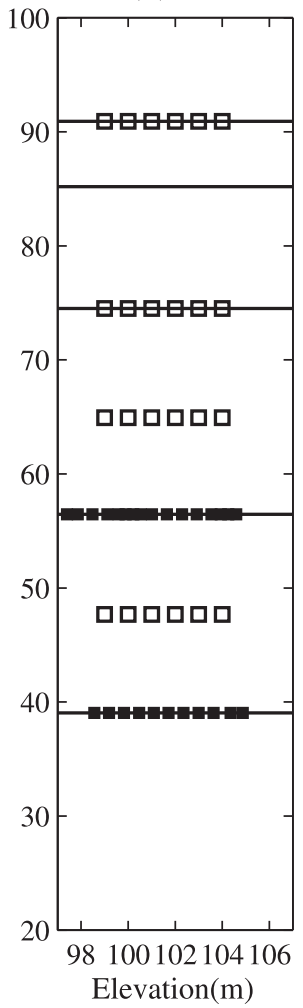

Figure 5. Locations of flowmeter wells (labeled as EBF Well), non-flowmeter wells (labeled as nonEBF Well), additional anchors, and cross sections showing anchor placement. (a) Plan view, (b) vertical cross section along $\mathrm{T} 1$, and (c) vertical cross section along T2. The universal coordinate system was rotated $35^{\circ}$ clockwise to make the rotated $y$-axis align with the major flow direction.

and conditional covariance $\eta^{2} \mathbf{R}_{\boldsymbol{\vartheta}_{b} \mid \boldsymbol{\vartheta}_{a}}$, where $\mathbf{R}$ denotes the correlation matrix with each element representing a twopoint spatial correlation. The conditional mean and correlation matrix are obtained as,

$$
\begin{aligned}
& \mu_{\boldsymbol{\vartheta}_{b} \mid \boldsymbol{\vartheta}_{a}}=\beta+\mathbf{R}\left(\mathbf{x}_{b}, \mathbf{x}_{a}\right) \mathbf{R}\left(\mathbf{x}_{a}, \mathbf{x}_{a}\right)^{-1}\left(\boldsymbol{\vartheta}_{b}-\beta\right), \\
& \mathbf{R}_{\boldsymbol{\vartheta}_{b} \mid \boldsymbol{\vartheta}_{a}}=\mathbf{R}\left(\mathbf{x}_{b}, \mathbf{x}_{b}\right)+\mathbf{R}\left(\mathbf{x}_{b}, \mathbf{x}_{a}\right) \mathbf{R}\left(\mathbf{x}_{a}, \mathbf{x}_{a}\right)^{-1} \mathbf{R}\left(\mathbf{x}_{a}, \mathbf{x}_{b}\right),
\end{aligned}
$$

where $\mathbf{x}_{a}$ and $\mathbf{x}_{b}$ are locations of type-A and type-B anchors, respectively, $\mathbf{R}\left(\mathbf{x}_{a}, \mathbf{x}_{b}\right)$ is the cross-correlation matrix between $\boldsymbol{\vartheta}_{a}$ and $\boldsymbol{\vartheta}_{b}$, and $\mathbf{R}\left(\mathbf{x}_{a}, \mathbf{x}_{a}\right)$ and $\mathbf{R}\left(\mathbf{x}_{b}, \mathbf{x}_{b}\right)$ are the autocorrelation matrices for $\boldsymbol{\vartheta}_{a}$ and $\boldsymbol{\vartheta}_{b}$, respectively. When the field is not multivariate normal, normal transform techniques, such as box-cox transformation [Box and Cox, 1964], could be applied as a first step.
[27] In summary, the prior distribution of the parameters is

$p\left(\boldsymbol{\theta}, \boldsymbol{\vartheta}, \mathbf{K}_{A} \mid \mathbf{z}_{\mathrm{EBF}}, \mathbf{z}_{\mathrm{IJNJ}}\right) \propto p\left(\boldsymbol{\vartheta}_{b} \mid \boldsymbol{\theta}, \boldsymbol{\vartheta}_{a}\right) p\left(\boldsymbol{\theta}, \boldsymbol{\vartheta}_{a}, \mathbf{K}_{A} \mid \mathbf{z}_{\mathrm{EBF}}, \mathbf{z}_{\mathrm{INJ}}\right)$.

\subsubsection{Field Generation}

[28] We generated random fields conditioned on the prior distribution of structural parameters and anchors. We first generated unconditional fields given structure parameters using a fast Fourier transform method [Nowak et al., 2003], and conditioned the fields on anchors using kriging [Rubin, 2003]. In this study, we also have a joint distribution of depth-average conductivities $\left(\mathbf{K}_{A}\right)$ at 10 wells without flowmeter data from assimilating the constant-injection tests. These data have support volumes as large as the saturated portion of the well and they could not be directly 
used in conditional 3-D field generation as anchors. However, they were included as useful prior information, as shown in Appendix, through a procedure that connects data with different support volumes.

\subsubsection{Forward Simulation}

[29] We simulated the flow and transport processes using PFLOTRAN. This code was chosen for its unique capability of simultaneously running multiple realizations on a supercomputer, which enabled us to complete the simulations on hundreds of thousands of random fields within a reasonable time frame on the Franklin supercomputer at the National Energy Research Scientific Computing Center (NERSC).

[30] According to Hammond and Lichtner [2010], the governing flow equations in PFLOTRAN are based on the Richards equation with velocities calculated by Darcy's law. For flow in the unsaturated zone, the van Genuchten model [van Genuchten, 1980] is used to relate capillary pressure to water saturation and the Burdine relation [Burdine, 1953] is used for the relative permeability function. The transport processes considered for the nonreactive tracer include advection, dispersion, and diffusion. The code employs the finite-volume method to discretize the governing equations and solve the flow and transport equations sequentially, i.e., passing water density, saturation state, and velocity field from the flow to the transport equations at each time step.

[31] The domain of simulation measures $122 \mathrm{~m} \times 122 \mathrm{~m} \times$ $10 \mathrm{~m}$ in size, which encapsulates the well field as shown in Figure 2. The base of the model domain lies at $97 \mathrm{~m}$ elevation above sea level, which corresponds to the lowest level of the screen among all of the monitoring wells. The top of the model is chosen to cover the maximum water level during the entire simulation period. The grid size is $2 \mathrm{~m}$ in the horizontal plane and $0.5 \mathrm{~m}$ in the vertical, and this resolution is considered sufficient for expected horizontal and vertical scales.

[32] We used transient hydrostatic head boundary conditions on the four lateral planes of the domain boundary, as the observed head showed significant variations during the experiment. In order to obtain the transient head values at every time step, we first fit a plane to hourly water level data at three corner wells within the IFRC well field (wells 2-22, 2-26, and 3-24), and then used the linear plane equation to extrapolate the water level to the corner points of the model domain, which was used as the hydrostatic head boundary condition during simulations. Considerable uncertainty may exist in the head boundary conditions estimated through triangulation. However, there was no better alternative at the time this study was carried out.

[33] We employed no-flux boundary conditions for the top and bottom boundaries, as the recharge at the top was relatively small [Rockhold et al., 2009] and the bottom of the modeling domain was constrained by the fine-grained Ringold formation. Initial flow conditions were specified as hydrostatic head corresponding to a water table at $105 \mathrm{~m}$.

[34] For modeling the tracer transport, we used zero tracer concentration as the initial condition over the entire model domain. The initial concentration was maintained at all boundaries except the top and bottom boundaries, where no-flux boundary conditions were specified.
[35] We used $1 \mathrm{~h}$ as the maximum time step in the simulation, and the PFLOTRAN program automatically reduced the time step when needed to meet accuracy requirements. The simulation was run up to $250 \mathrm{~h}$, at which the bromide breakthrough concentration in most of the wells was completed.

[36] We put multiple observation points at discrete depths along the wells that are fully screened over the saturated zone; we then calculated flux-averaged concentrations to represent the average concentration at each well.

\subsubsection{Likelihood Calculation}

[37] The likelihood function in MAD is a multidimensional probability density of the observations, evaluated at the observed data, $p\left(\mathbf{z}_{\text {TRC }} \mid \boldsymbol{\theta}, \boldsymbol{\vartheta}_{a}, \mathbf{K}_{A}\right)$, where the vector $\mathbf{z}_{\text {TRC }}$ (which is the tracer data to be assimilated), consists of time series of breakthrough concentrations in multiple wells. We based the likelihood calculation on an ensemble of tracer breakthrough curves conditioned on the parameters: For a given realization of parameters $\left\{\boldsymbol{\theta}, \boldsymbol{\vartheta}_{a}, \mathbf{K}_{A}\right\}$, we generated 400 conditional random fields and provided them to PFLOTRAN for the flow and transport simulation. The simulated breakthrough curves obtained from each random field constitute one realization of $\mathbf{z}_{\mathrm{TRC}}$, and the ensemble of 400 realizations constitutes the sample pool for estimating the likelihood as illustrated in Figure $6 \mathrm{a}$ for an individual well.

[38] The spatial-temporal concentration data has a very high dimension (in hundreds), which is not manageable in a nonparametric density estimation [Scott and Sain, 2004]. We therefore computed the temporal moments [Cirpka and Kitanidis, 2000a, 2000b] of the breakthrough curve at each well to be used instead of the complete time series. This reduces the dimension of the data vector and hence of the likelihood function, at the expense of a loss in detail. We used only one of the temporal moments at a time, and we compared the performance of inversion conditioned on the zeroth- and first-order moments and on the normalized first-order temporal moment.

[39] The $k$-th temporal moment of the breakthrough curve is defined as,

$$
m_{k}=\int_{0}^{\infty} t^{k} c(t) d t
$$

where $c(t)$ is the concentration measured at time $t$. In calculating the temporal moments on the observed and simulated breakthrough curves at each well, we approximate them using the trapezoidal rule, i.e.,

$$
m_{k}=\sum_{i=1}^{n-1} 0.5\left(c_{i}+c_{i+1}\right)\left(t_{i+1}^{k}-t_{i}^{k}\right)
$$

where $c_{1}, \ldots, c_{n}$ are concentrations measured at times $t_{1}, \ldots, t_{n}$, respectively.

[40] The zeroth temporal moment $\left(m_{0}\right)$ and normalized first-order temporal moment $\left(m_{1} / m_{0}\right)$ represent the mass recovery and mean arrival time at an observation point, respectively. By conditioning on $m_{0}$, the important information is whether the observation point has seen the right 
(a)



(b)

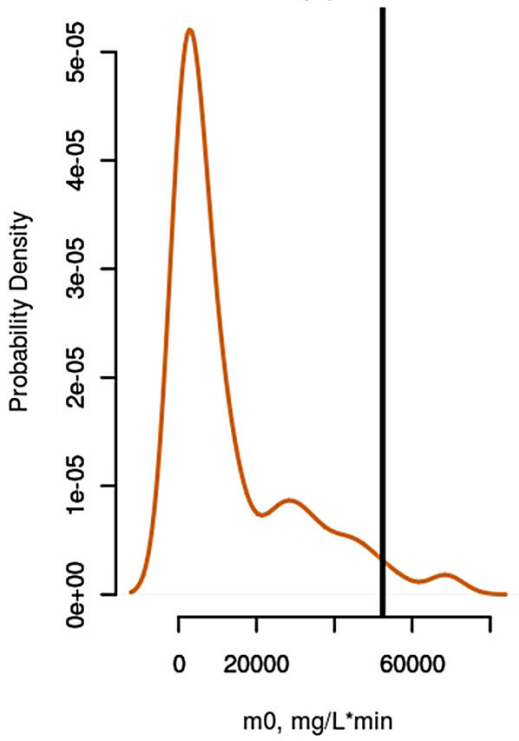

(c)



Figure 6. Illustration of likelihood calculation for one conditioning well. (a) Tracer concentrations normalized by the injection concentration $C_{0}$. The circle scatters are the observed breakthrough data and the lines represent ensemble of simulated breakthrough curves. $(b, c)$ The probability density functions of the 0 -th and first temporal moments estimated from the ensemble of simulated breakthrough curves in Figure $6 \mathrm{a}$. The vertical lines in Figures $6 \mathrm{~b}$ and $6 \mathrm{c}$ represent the corresponding temporal moments calculated from the observed breakthrough curve, and the $y$-coordinates of their intersections with the curve are the estimated likelihood, depending on which moment is used for conditioning.

amount of tracer mass within a specified time frame; by conditioning on $m_{1} / m_{0}$, the emphasis is on the right timing without considering the mass recovery.

[41] Assuming that we have selected the $k$-th temporal moments at $p$ wells for conditioning, the data for likelihood calculation is then $\mathbf{z}_{\mathrm{TRC}}=\left\{m_{\mathrm{k}}^{1}, m_{\mathrm{k}}^{2}, \ldots, m_{\mathrm{k}}^{p}\right\}$, where the superscripts represent the well indices and the subscripts represent the order of the moment used for representing the breakthrough curve. Since each random field produces one realization of $m_{\mathrm{k}}^{1}, m_{\mathrm{k}}^{2}, \ldots, m_{\mathrm{k}}^{p}$, there are 400 such realizations for each parameter set. The likelihood calculation is then a $p$-dimensional joint probability density estimation problem with 400 samples. Figures $6 \mathrm{~b}$ and $6 \mathrm{c}$ demonstrate the probability densities of $m_{0}$ and $m_{1}$ for an individual well, estimated from the ensemble of the breakthrough curves at the same well as shown in Figure 6a. If there is only one well used for conditioning, the likelihood is then the probability density evaluated at the observed $m_{0}$ or $m_{1}$, or some other forms of temporal moments. Although we used a one-well case for illustration in Figure 6, the same approach is applied in the likelihood calculation when more than one well is used for conditioning.

[42] While there are various techniques available for multidimensional density estimation [Scott and Sain, 2004], kernel density estimator has widely been adopted as a powerful nonparametric tool. Thus, we adopted the kernel density estimator for the likelihood calculation and used an $\mathrm{R}$ package, $\mathrm{np}$ [Hayfield and Racine, 2008], for the computation. The details of the algorithm implemented in the $\mathrm{np}$ package are available in the work of Hayfield and Racine [2008]. We performed the likelihood calculation for each random draw of parameters from the prior distribution.

\subsection{Posterior Distribution of Parameters}

[43] The posterior distribution of parameters, including structural parameters and anchors, following the assimilation of the tracer test is derived through Bayes' rule:

$$
\begin{aligned}
& p\left(\boldsymbol{\theta}, \boldsymbol{\vartheta} \mid \mathbf{z}_{\mathrm{EBF}}, \mathbf{z}_{\mathrm{IJNJ}}, \mathbf{z}_{\mathrm{TRC}}\right) \propto p\left(\mathbf{z}_{\mathrm{TRC}} \mid \boldsymbol{\theta}, \boldsymbol{\vartheta}, \mathbf{K}_{A}\right) p\left(\boldsymbol{\vartheta}_{b} \mid \boldsymbol{\theta}, \boldsymbol{\vartheta}_{a}, \mathbf{K}_{A}\right) \\
& \quad p\left(\boldsymbol{\theta}, \boldsymbol{\vartheta}_{a}, \mathbf{K}_{A} \mid \mathbf{z}_{\mathrm{EBF}}, \mathbf{z}_{\mathrm{INJ}}\right),
\end{aligned}
$$

where we assume that $\left\{\boldsymbol{\theta}, \boldsymbol{\vartheta}_{a}, \mathbf{K}_{A}\right\}$ captures all of the relevant information that could be retrieved from the flowmeter and constant-rate injection tests.

[44] The posterior distribution of parameters can be derived using sample-based methods such as the Markov chain Monte Carlo (MCMC) method [Andrieu et al., 2003] and sequential Monte Carlo method (i.e., particle filter) [Moradkhani et al., 2005], which are widely adopted in hydrology literature when the analytical form of distribution is not available.

\section{Results and Discussions}

[45] This section presents inversion results conditioned on the tracer data from both a synthetic study that mimics the actual conditions at the Hanford IFRC site and the implementation of MAD on the March 09 tracer test. We start this section by presenting a set of wells that were used for conditioning and testing, respectively. The conditioning set provides observed data to the inversion process, and it is equivalent to the training set in a traditional model calibration effort. The testing set is used to test the predictive performance of the inversion product when the true values of parameters are not available in real field applications. 


\subsection{Wells Used in Assimilation}

[46] We selected a set of conditioning wells (2-07, 2-26, 2-27, 2-28, 2-17, 2-19, 3-30, 3-32, and 2-29), for likelihood calculation. As shown in Figure 7, this set of wells was balanced for both longitudinal and transverse coverage. A large portion of wells was selected from the depth-discrete wells clusters in order to minimize the negative influence of vertical wellbore flow on inversion and to capture the vertical heterogeneity profile as well. The testing wells used in the real field case (2-08, 2-11, and 3-28), are also shown. The fully screened wells used in conditioning or testing (2-07, 2-17, 2-19, 2-08, 2-11, and 3-28) were chosen in consideration of spatial coverage, and a relatively flatter permeability vertical profile as revealed by the flowmeter tests, which may lead to less significant vertical wellbore flow. Although the vertical wellbore flow does not exist in the synthetic case, we used the same set of conditioning wells for consistency.

\subsection{Synthetic Study}

[47] Several complex conditions encountered in the field tracer test, such as vertical wellbore flow and transient flow boundary conditions induced by the adjacent Columbia River, were difficult to quantify in the numerical model. Since they could adversely affect the performance of the MAD framework in addressing the parameter uncertainty, we performed a synthetic study, constructed to have similar features to the actual site, including the conditions prevailing during the March 09 tracer test. The synthetic case study was also used to assess the extent of adverse effects caused by the complex flow conditions.

[48] In the synthetic study, we first generated a reference 3-D hydraulic conductivity field using a parameter set generated from the prior distribution. We then ran a forward simulation on the reference field, using PFLOTRAN with the same set of flow and transport conditions as the one used in assimilating the actual data. The forward simulation provided the tracer breakthrough curves at the wells that were monitored in the actual tracer test. Random Gaussian noises (zero mean and standard deviation set at $5 \%$ of the measured values) were added to the simulated breakthrough data as measurement errors. This set of the synthetic tracer breakthrough curves was used as if we had known all of the field test conditions precisely (i.e., the transient flow boundary conditions, the depth to the Hanford-Ringold interface, and no vertical wellbore flow).

[49] We studied the ability of the MAD framework to capture the true geostatistical structural parameters (i.e., mean, variance, vertical, and horizontal scales), conditioning on different temporal moments. Figure 8 shows the prior and posterior distributions of the structural parameters conditioned on $m_{0}, m_{1}$, and on their ratio $m_{1} / m_{0}$. The prior

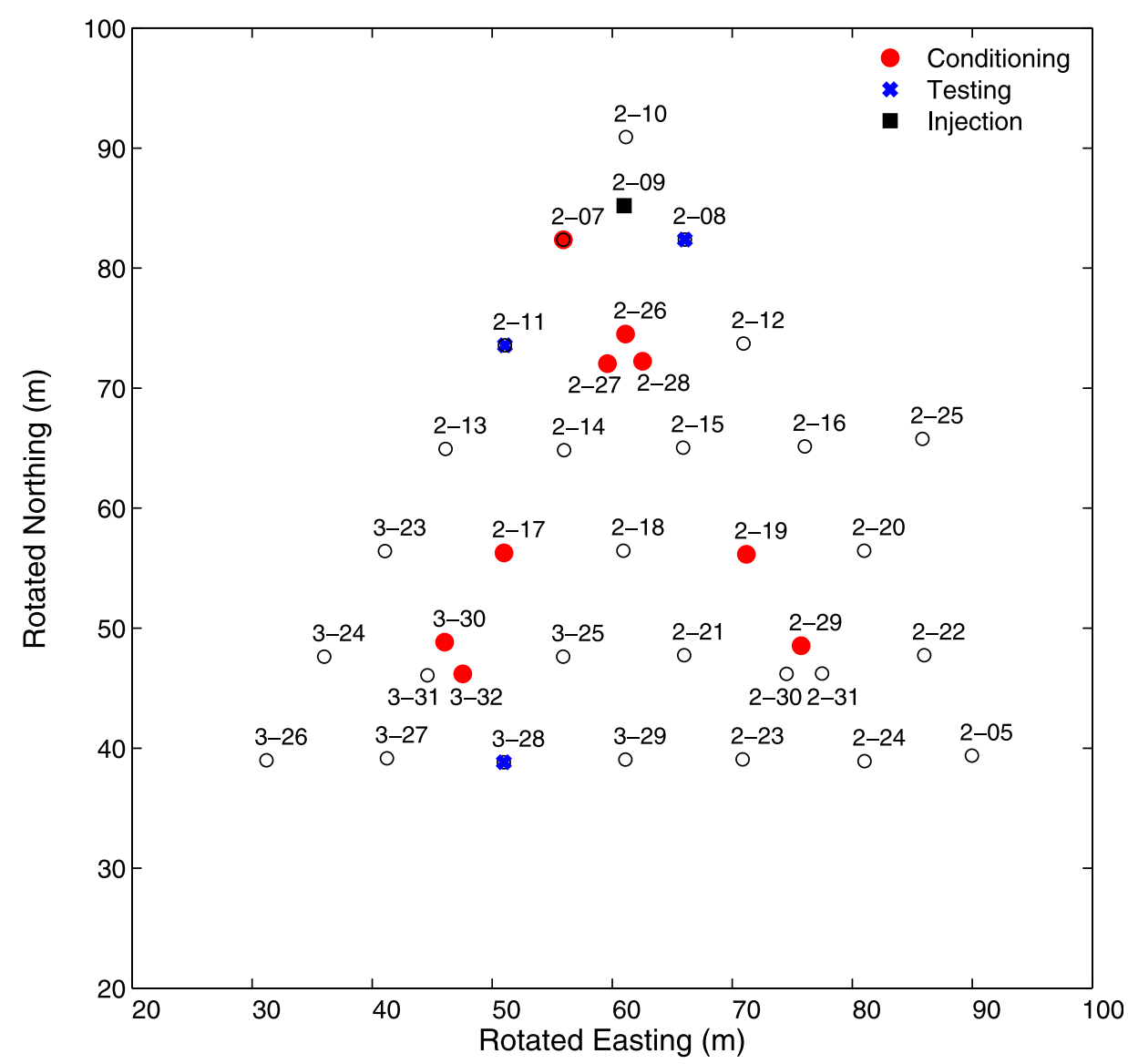

Figure 7. Wells selected for conditioning and validation in assimilating the tracer test data. The universal coordinate system was rotated $35^{\circ}$ clockwise to make the rotated $y$-axis align with the major flow direction. 
Mean



Horizontal Scale

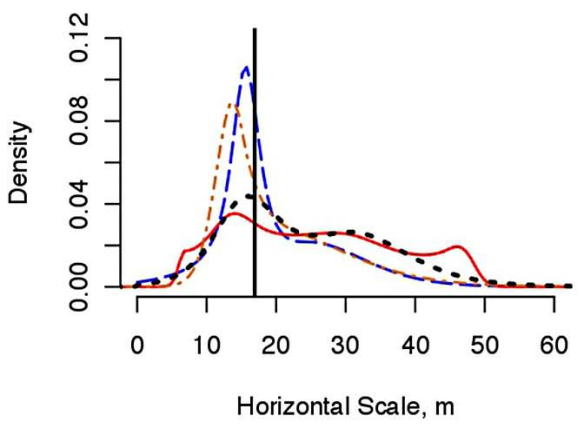

Variance

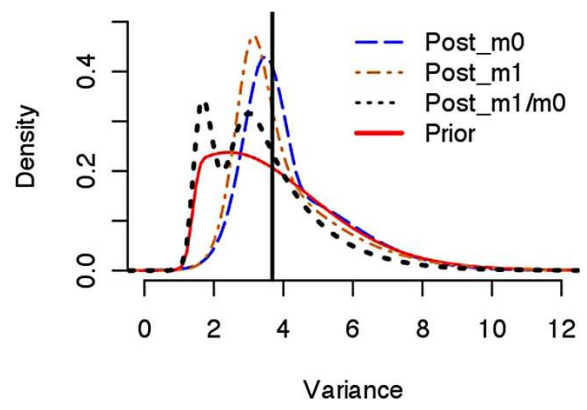

Vertical Scale

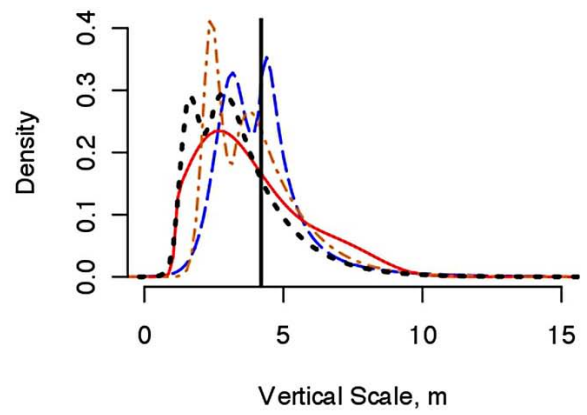

Figure 8. Marginal prior and posterior distributions of the structural parameters for $\ln \mathbf{K}$ in the synthetic study (the vertical lines are the true parameter values used to generate the reference field).

distributions of the 3-D structural parameters were inferred from the distributions of depth-discrete hydraulic conductivities at the flowmeter wells using the Bayesian modelbased geostatistics [Diggle and Ribeiro, 2006]; details are available in the work of Murakami et al. [2010]. The posterior distributions of the structural parameters (Figure 8) after conditioning on the tracer data show convergence of the modes toward the actual values and reduction in uncertainty as manifested by the narrower distributions. Among the posterior distributions conditioned on different temporal moments, conditioning on $m_{0}$ captures the true structural parameters the best (in section 4.4, we will discuss the relative performance of different temporal moments in characterizing spatial heterogeneity under temporally fluctuating flow conditions after presenting the results from the field case study). Although there is little change in the probability distributions of the field mean after conditioning on the tracer data, considerable improvement is seen in the variance, horizontal scale, and vertical scale, in terms of reduction in uncertainty or proximity of the mode to the true value. The significant improvement in the structural parameter estimation demonstrates the effectiveness of MAD in identifying the heterogeneity structure of the hydraulic conductivity field, conditioned on the temporal moments of tracer breakthrough data.

[50] We also examined how local heterogeneity was captured with and without tracer data assimilation. We selected two transects, shown as T1 and T2 in Figure 5, to demonstrate the mean values and confidence intervals of vertical hydraulic conductivity profiles obtained with and without using the tracer data. To compute the mean values and confidence intervals, we generated 500 realizations of parameter sets from the prior and posterior distributions, respectively, and 20 random fields from each parameter set, which produced 10,000 prior and posterior realizations of hydraulic conductivity profiles at each selected well.

[51] The plots in Figures 9 and 10 show the mean values and $95 \%$ confidence intervals of the hydraulic conductivity profiles along the selected wells, conditioned on $m_{0}$ of the conditioning wells. The other two forms of the first-order temporal moments were found to produce inferior results, and therefore they are not shown here (we will discuss the relative performance of different temporal moments in characterizing spatial heterogeneity under temporally fluctuating flow conditions after presenting the results from the field case study). We note that the true profiles at the flowmeter wells (e.g., 2-18, 3-29, 3-24, 3-25, and 2-21) were captured more accurately after conditioning on the tracer data, judging from both the mean and 95\% confidence intervals. The improvement at the nonflowmeter wells (e.g., 2-10, 2-09, 2-26, 3-30, 2-19, and 2-22) or at the additional anchor locations was not as significant, although we still see some improvement as the mean estimates shift closer to the true value or uncertainty reduced. This difference is attributed to our assumption in this synthetic study such that the flowmeter wells have uncertainty only in the average hydraulic conductivity (or transmissivity), since we consider the normalized hydraulic conductivity profiles to be accurate. This setting provided more constraints to the inversion and has resulted in reduced uncertainty at the flowmeter wells. On the other hand, there was no equivalent information available at the nonflowmeter wells, thus there was larger uncertainty associated with inversion at the nonflowmeter wells. The effect of increased constraint 

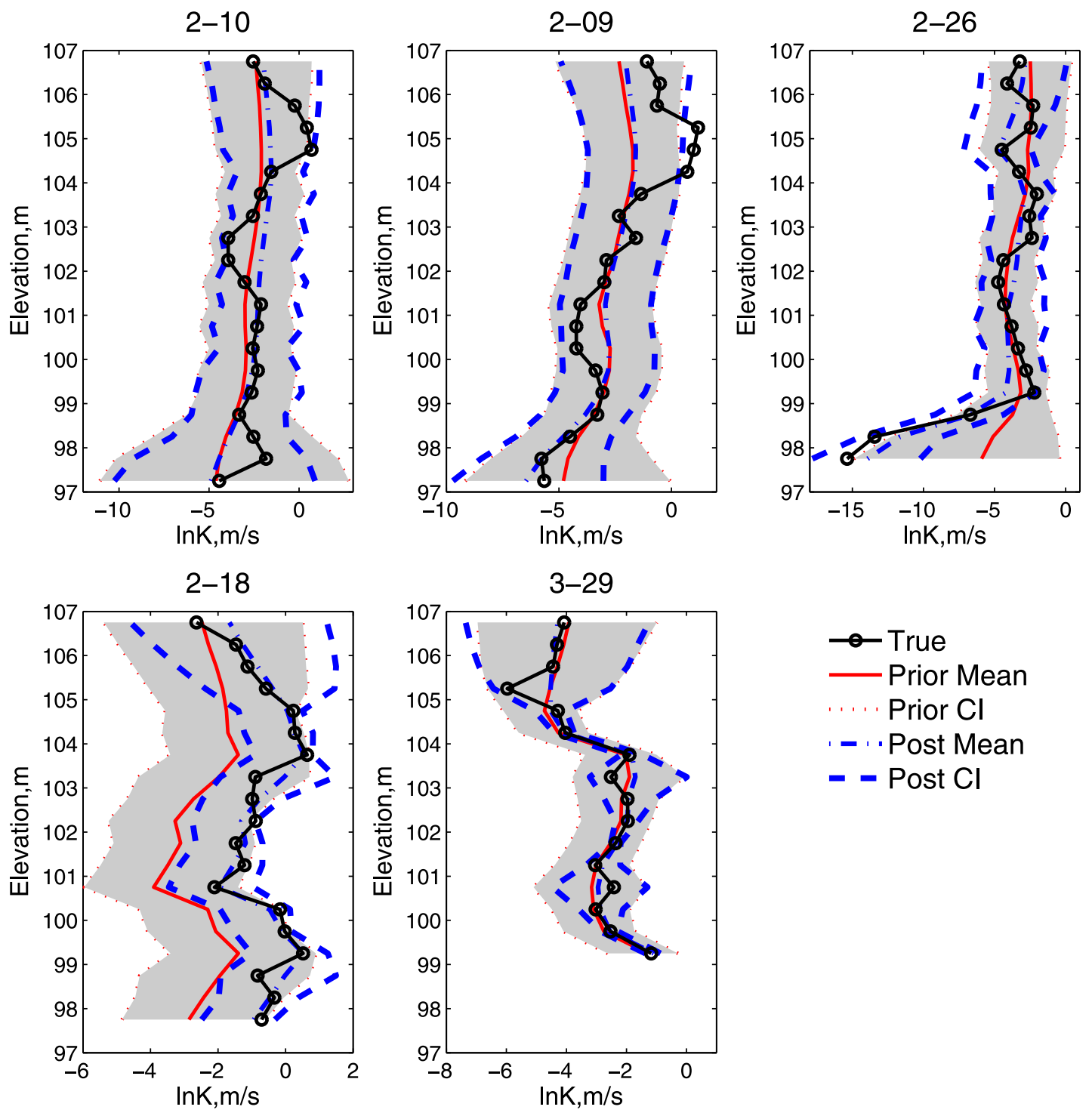

Figure 9. Hydraulic conductivity profiles in selected wells along transect T1 prior and posterior to conditioning on $m_{0}$. The area filled in gray is the predicted confidence interval before conditioning on the tracer data. Wells 2-18 and 3-29 are flowmeter wells.

is also seen at the nonflowmeter wells: for example, by comparing the estimated profile at well 2-26 versus that of well 2-09, or well 2-29 versus well 2-22, we found that the nonflowmeter wells with more neighboring flowmeter wells benefit more from the tracer experimental data due to the stronger conditioning effects from the type-A anchors.

\subsection{Implementation of MAD on the March 09 Tracer Test}

[52] It was evident from the synthetic study that the MAD technique is capable of capturing the aquifer heterogeneity from the tracer test given appropriate knowledge of the flow and transport conditions. Therefore, we proceeded on to assimilate the actual data from the March 09 tracer test, conditioned on the zeroth or the first temporal moments of the same set of conditioning wells used in the synthetic study.
[53] Figure 11 shows the marginal prior and posterior distributions of the geostatistical structural parameters. We note that conditioning on the mean arrival time leads to the largest reduction in uncertainty among the three choices of temporal moments, while conditioning on $m_{0}$ or $m_{1}$ tend to produce bimodal posterior distributions for the structural parameters. It is common for all three forms of temporal moments that the variance and vertical scale are shifted toward the higher values after conditioning on the tracer data, which may possibly imply better detection of contrast between more permeable and less permeable layers at the site.

[54] Bivariate density contours of the structural parameters are given in Figure 12, which shows the multiple modes of bivariate posterior distributions conditioned on $m_{0}$ or $m_{1}$, while those conditioned on $m_{0} / m_{1}$ are unimodal. The bivariate plots also reveal positive correlations between horizontal and vertical scales and variance, and 

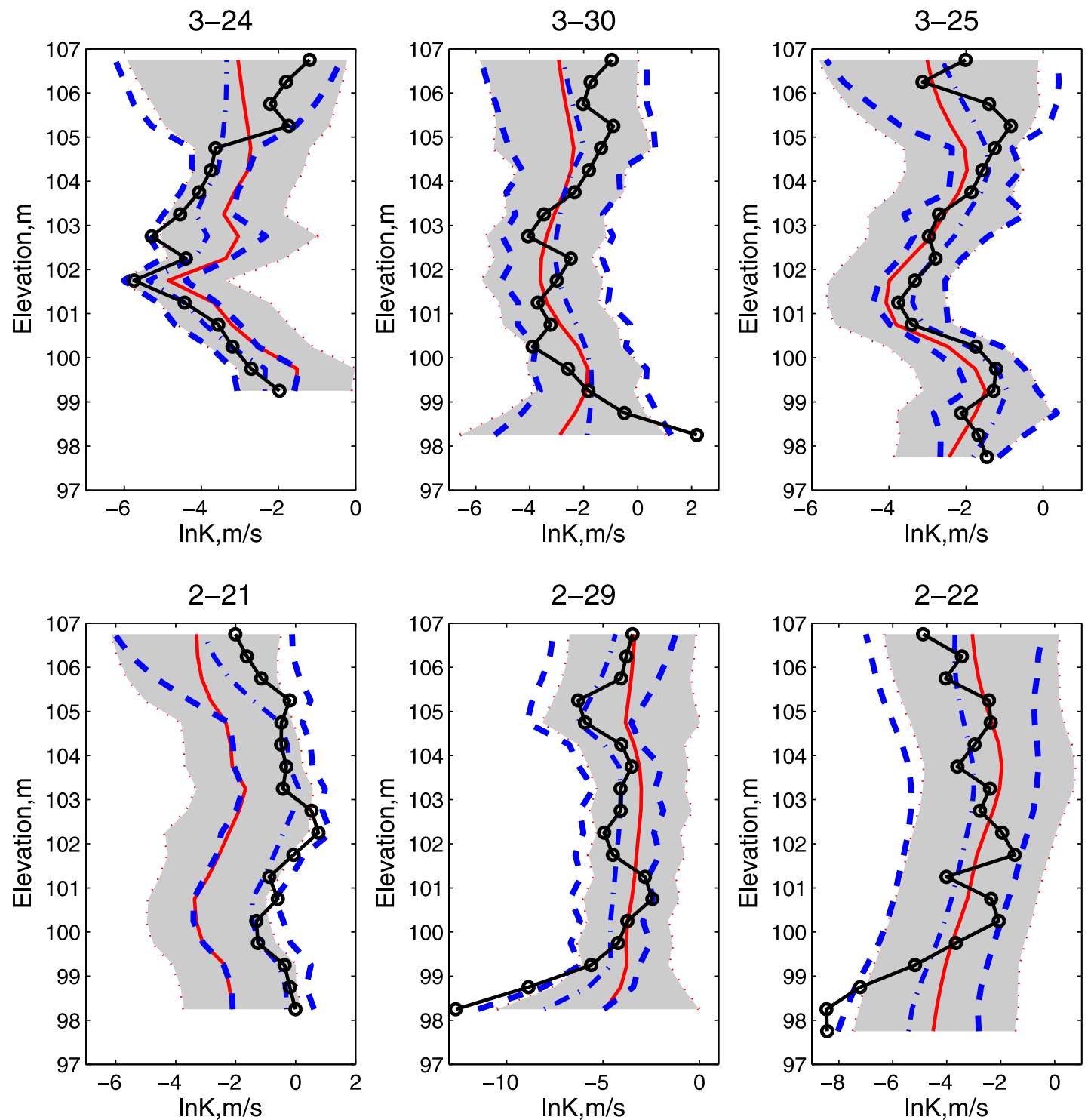

Figure 10. Hydraulic conductivity profiles in selected wells along transect T2 prior and posterior to conditioning on $m_{0}$. The area filled in gray is the predicted confidence interval before conditioning on the tracer data. Wells 3-24, 3-25, and 2-21 are flowmeter wells. Legend is the same as in Figure 9.

negative correlations between the mean and other structural parameters.

[55] Although the structural parameters are important for describing the spatial pattern of heterogeneity, connectivity of low permeability zones or high permeability zones of porous media is more important for flow and transport processes [Zinn and Harvey, 2003]. To evaluate the connectivity, we computed a mean field from 1000 random field realizations conditioned on a parameter set that yields the highest likelihood among all of the realizations of parameter sets (equivalent to a maximum likelihood estimator) for each temporal moment. The results based on the maximum likelihood (ML) estimator are shown to demonstrate what connectivity pattern each temporal moment favors.

[56] Figure 13 shows the vertical planes of mean $\log$ conductivity field at the cross-section of $\mathrm{T} 1$, conditioned on prior distribution of parameters and on the ML estimators of parameters based on different temporal moments of tracer data. Conditioning on $m_{0}$ or $m_{1} / m_{0}$ resulted in higher conductivity in shallow and deep layers near well 2-18 along transect $\mathrm{T} 1$, compared to the prior mean field. On the other hand, conditioning on $m_{1}$ leads to a more connected low-permeability zone in the intermediate depth. Figure 14 shows horizontal planes at three different elevations to demonstrate horizontal connectivity conditioned on the temporal moments of the tracer data. We can see that conditioning on $m_{0}$ resulted in low permeability close to boundaries and a more connected inner high-permeability zone, whereas conditioning on $m_{1}$ yields a more connected lowpermeability zone that extends to boundaries at different depths. Conditioning on $m_{1} / m_{0}$ generates the mean field that has more connected high-permeability zones, extending laterally to the boundaries. 

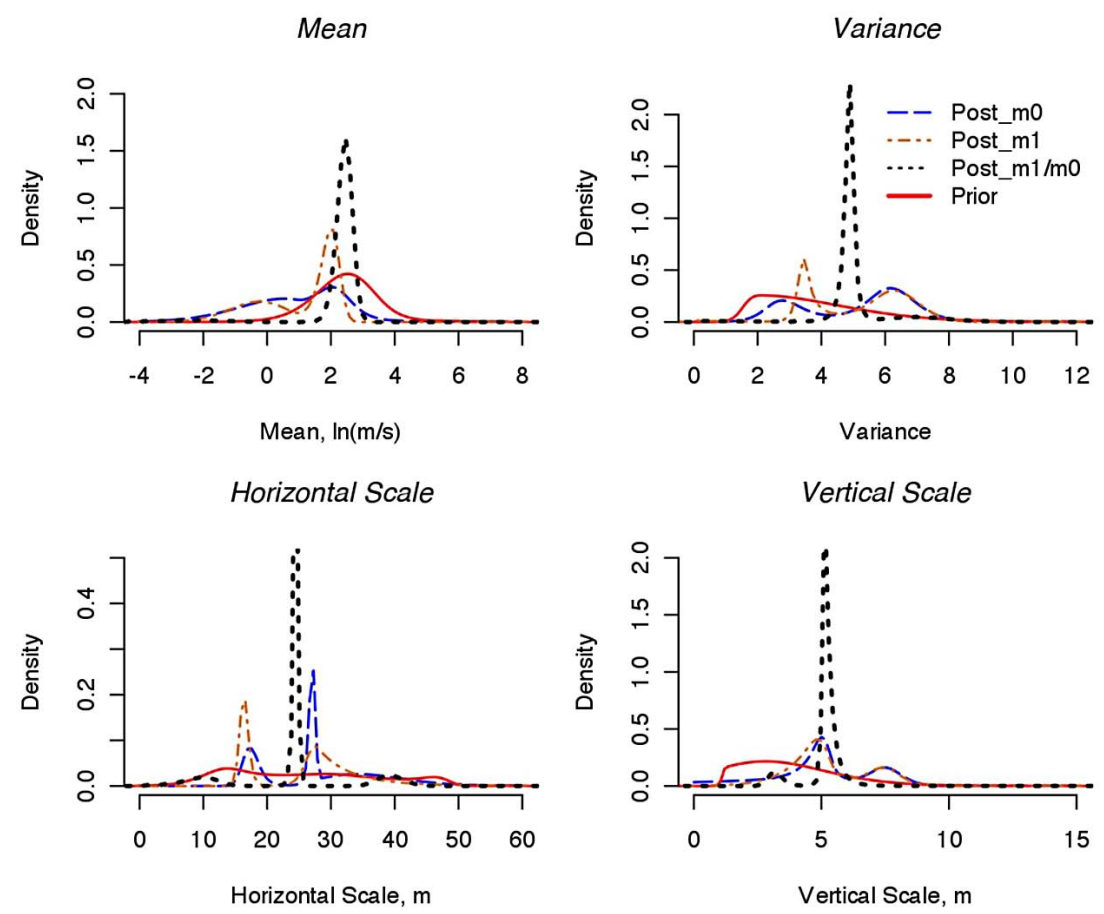

Figure 11. Marginal distributions of the structural parameters for $\ln \mathbf{K}$ at the IFRC site prior and posterior to conditioning on tracer data.

[57] With no true structural parameters or the true conductivity field to compare with in this application, it is difficult to assess the performance of our inversion. We therefore employed an alternative metric, based on the predicted tracer breakthrough curves in selected testing wells. We computed breakthrough curves at each well on 210,000 realizations of random hydraulic conductivity fields, with 100 fields generated from each of 2100 prior or posterior parameter sets. We then calculated the root-mean-square error (RMSE) for each simulated breakthrough curve with respect to the observed curve at a testing well, which is

$$
\sqrt{\sum_{i=1}^{n}\left(\frac{c_{s i m, i}}{c_{0}}-\frac{c_{o b s, i}}{c_{0}}\right)^{2} / n}
$$

with $c_{s i m, i}$ and $c_{o b s, i}$ being the simulated and observed concentrations at the $i$-th time step, respectively, $c_{0}$ being the injection concentration, and $n$ being the total number of observations. Thus, there were 210,000 samples of RMSE for each well, from which a probability distribution of RMSE could be constructed.

[58] Figure 15 shows the cumulative distribution functions (CDF) of RMSE at the selected testing wells. Effective inversion is expected to yield more mass of RMSE close to zero, i.e., higher CDF value at low-end RMSE indicates a better match to the observed breakthrough curves. We observe in Figure 15 that conditioning on $m_{0}$ and $m_{1}$ produces better matches to the observed data than the prior at all three wells. However, the performance of conditioning on $m_{1} / m_{0}$ fluctuates among wells such that it yields the best posterior match at wells 2-8 and 3-28 but it also yields the worst posterior match at well 2-11. This observation is consistent with the narrower posterior distribution of parameters conditioned on $m_{1} / m_{0}$, since a narrow distribution is more probable of missing the target when there is a bias in estimation due to inaccuracies in the conceptual model or measurement errors. This problem can be more severe if an optimal parameter set instead of parameter distribution is used. As bias is expected in estimating spatial properties from inversion [Kitanidis, 1998], MAD embraces this bias with distributions of parameters.

\subsection{Discussions}

[59] One of the interesting problems in this study is which form of temporal moments of tracer breakthrough data is the most effective in capturing the spatial heterogeneity of the hydraulic conductivity. Although $m_{1} / m_{0}$ was found to be the most relevant to conductivity for a wide plume under steady state flow conditions [Nowak and Cirpka, 2006; Pollock and Cirpka, 2010], the information content in each temporal moment is more complicated for a small plume under dynamic flow conditions, which prevail at our study site. The flow and transport behavior through a porous medium under transient flow conditions is strongly influenced by the interactions between the spatial heterogeneity of conductivity and the temporal dynamics of the flow fields [Goode and Konikow, 1990; Cirpka and Attinger, 2003; Dentz and Carrera, 2005]. As a result, $m_{0}$ under these conditions could contain useful information for the meandering of the plume (hence the spatial heterogeneity of hydraulic conductivity), rather than being constant as in the case of a wide plume under steady flow conditions.

[60] In the synthetic study, conditioning on $m_{0}$ yielded the best estimate to the true parameters, on the other hand, in the real case, conditioning on $m_{1} / m_{0}$ yielded the most spiked posterior distributions for the structural parameters. A further examination on the mean field generated from the 

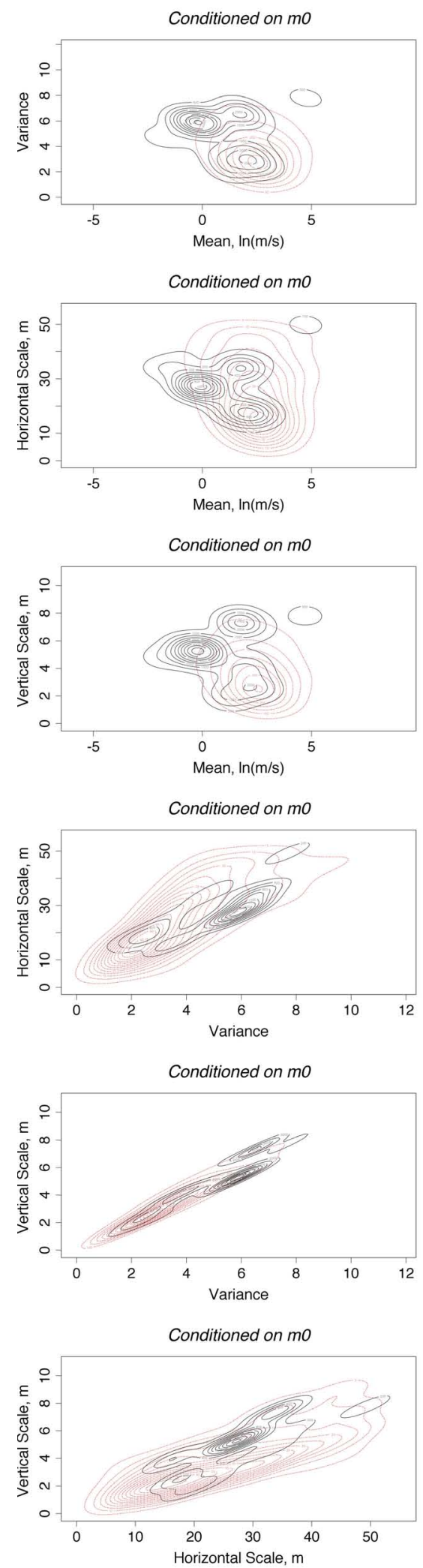
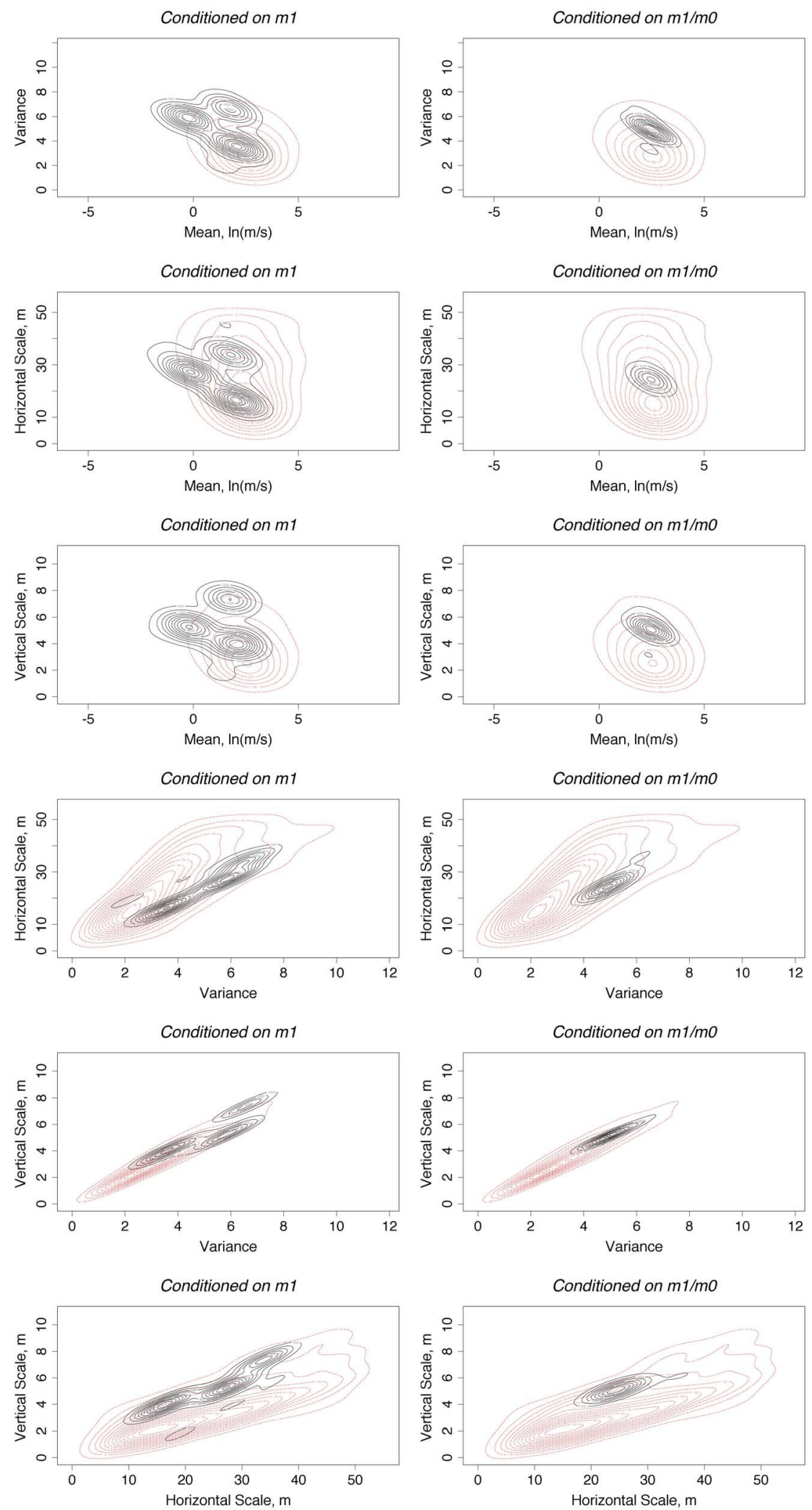

Figure 12. Bivariate distributions of the structural parameters for $\ln \mathbf{K}$ at the IFRC site prior and posterior to conditioning on tracer data. Solid lines are posterior distribution contours and dashed lines are prior distribution contours. 
(a) Mean Field: Prior

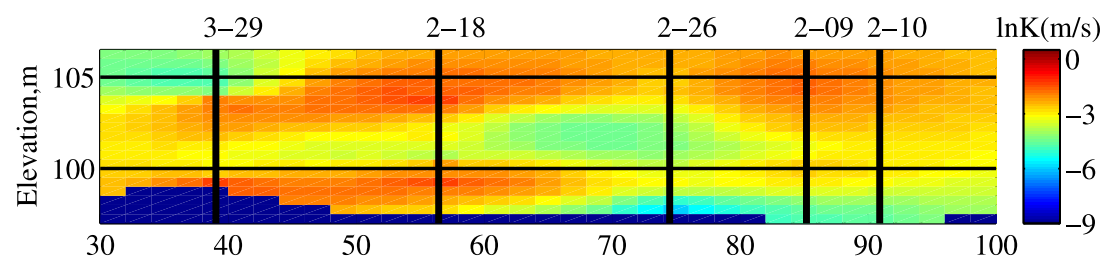

(b) Mean Field: Posterior, conditioned on $\mathrm{m} 0$



(c) Mean Field: Posterior, conditioned on $\mathrm{m} 1$

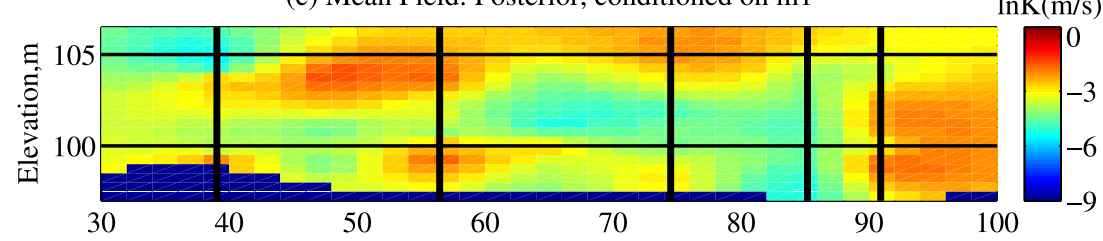

(d) Mean Field: Posterior, conditioned on $\mathrm{m} 1 / \mathrm{m} 0$



Figure 13. Mean log-conductivity field at the cross section along T1 prior and posterior to conditioning on tracer data for March 09 tracer test. (a) Mean field prior to conditioning on the tracer data. (b-d) Mean fields posterior to conditioning on $m_{0}, m_{1}$, and $m_{1} / m_{0}$, respectively. The posterior fields were generated using the ML estimator of parameters for each temporal moment.

ML estimator of parameters revealed that different connectivity patterns were favored when different forms of temporal moments were used for conditioning. Using the breakthrough curves at selected testing wells as an alternative metric to evaluate the relative performance of temporal moments in the real case, we found that results based on $m_{1} / m_{0}$ could lead to the best match in some wells while providing the worst match in some other wells, as a result of the narrower posterior distribution of the parameters. It is expected that the relative performance of temporal moments depend not only on the set of wells that were selected for conditioning but also on the wells that were used for testing. Therefore, it may be necessary in real applications to compare the performance of different temporal moments and experiment with different sets of conditioning and testing wells. However, the results from an alternative set of conditioning and testing wells were not available in this study because we have a limited choice of wells due to the wellbore flow complications.

\section{Conclusions}

[61] We presented in this study a method for improving site characterization using data from tracer experiments, in addition to data from constant-rate injection tests and flowmeter tests. The proposed procedure was based upon a Bayesian data assimilation technique, MAD, which enables assimilation of various types of data collected at multiple scales. We used the hydraulic conductivity field characterized with constant-injection tests and flowmeter tests [Murakami et al., 2010] as the prior information, and the posterior field were inferred conditioning on the temporal moments of tracer data at a set of wells. The MAD technique is computationally intensive and it was only made possible with the availability of high-performance computing codes, such as PFLOTRAN, and supercomputer resources.

[62] The proposed method was verified using a synthetic study before being applied to the real tracer test data. The synthetic study showed the effectiveness of the proposed method in capturing the true underlying heterogeneity of the hydraulic conductivity field, in the absence of conceptual model errors. We also found in the synthetic study that the normalized hydraulic conductivity profile estimated from the flowmeter data alleviated the nonuniqueness in inversion and substantially reduced uncertainty in the estimated hydraulic conductivity through conditioning. The performance of the inversion in the real case study was assessed by the RMSE of simulated breakthrough curves with respect to the observed ones at selected testing wells. A reduction in RMSE was observed after inversion. 
(a) Prior

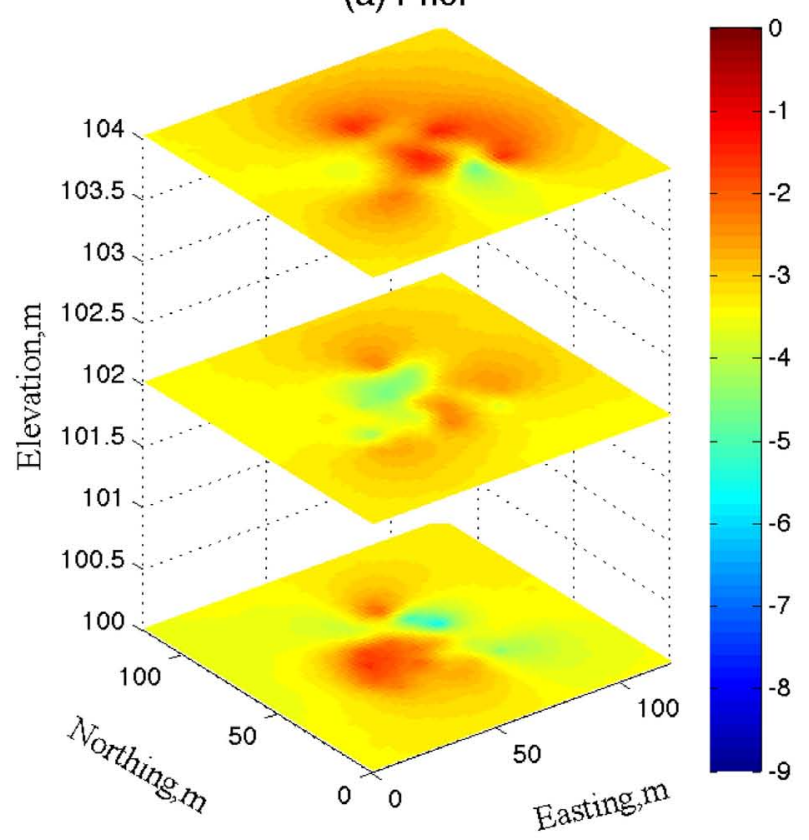

(c) Posterior conditioned on $\mathrm{m} 1$

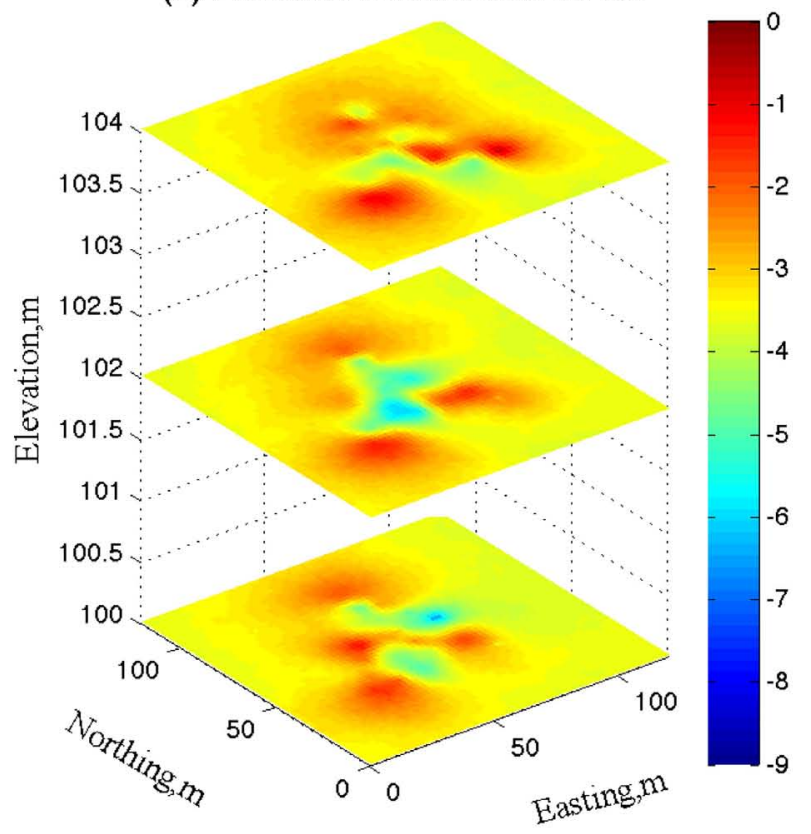

(b) Posterior conditioned on mo

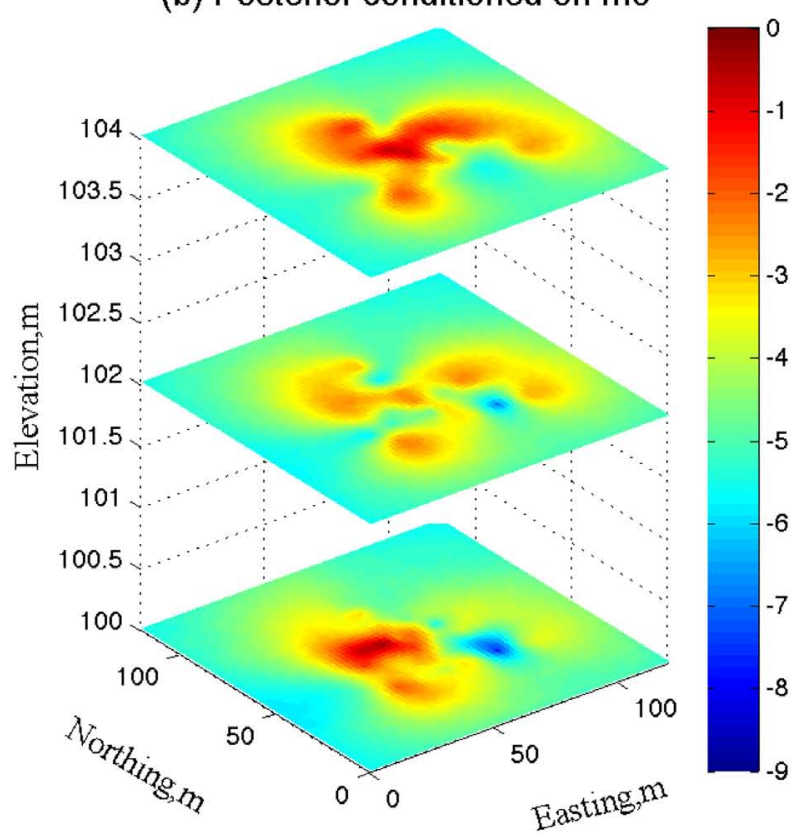

(d) Posterior conditioned on $\mathrm{m} 1 / \mathrm{mo}$

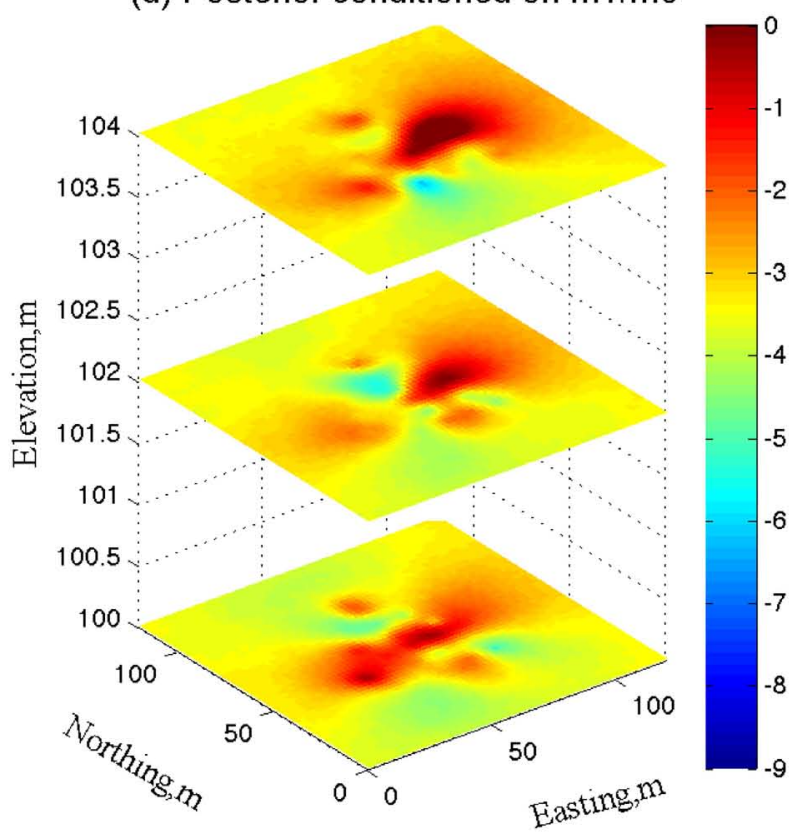

Figure 14. Mean log-conductivity field at the different elevations prior and posterior to conditioning on tracer data for March 09 tracer test. (a) Mean field prior to conditioning on the tracer data. (b-d) Mean fields posterior to conditioning on $m_{0}, m_{1}$, and $m_{1} / m_{0}$, respectively. The posterior fields were generated using the ML estimator of parameters for each temporal moment.

[63] A primary feature that distinguishes this study from others that involve inversion based on temporal moments is that our inversions were conducted under dynamic flow conditions. Therefore, the information content in each temporal moment is more complicated than in the steady state cases, since the flow and transport behavior through a porous medium under transient flow conditions is strongly influenced by the interactions between the spatial heterogeneity of conductivity and the temporal dynamics of the flow fields. We compared the relative performance of three forms of temporal moments, $m_{0}, m_{1}$, and $m_{1} / m_{0}$, in capturing the true heterogeneity in both the synthetic and real case studies. In the synthetic study, conditioning on $m_{0}$ yielded the best estimate of the true parameters. In the real case, conditioning on $m_{1} / m_{0}$ yielded the narrowest posterior distributions for the structural parameters, while conditioning on $m_{1}$ or $m_{0}$ yielded multimodal posterior distributions. It was found in the real case study that different connectivity 



Figure 15. Distribution of RMSE prior and posterior to conditioning on the March 09 tracer test data at selected wells. Wells 2-8, 2-11, and 3-28 were used for testing. The injection concentration is $c_{0}$.

patterns were favored when different forms of temporal moments were used for conditioning. It is expected that the relative performance of temporal moments could vary case by case, and therefore it may be necessary in real applications to compare the performance of different temporal moments and experiment with different sets of conditioning and testing wells.

[64] We expect the inversion results in the real case study to be negatively affected by uncertainties involved in the forward simulations, including but not limited to transient flow boundary conditions estimated through triangulation and vertical wellbore flow induced by the river stage fluctuations in the adjacent Columbia River. As an initial attempt to assimilate the real experimental data under the complex field conditions, the results from this study can be used to identify future research directions, which could include collecting more reliable flowmeter data (or equivalent type-A data) near the injection well or integrating other types of data such as borehole and tomographic geophysical data to provide better prior information on the vertical profile of hydraulic conductivity, and addressing the conceptual model uncertainty, such as uncertainties in boundary condition and vertical wellbore flow, in inversion.

\section{Appendix A: Including Depth-Averaged Conductivities in 3-D Field Generation}

[65] Figure A1 shows all the conditional information available for field generation, where $\boldsymbol{\vartheta}_{a}$ is a depth-discrete log conductivity value along each flowmeter well, $\boldsymbol{\vartheta}_{b}$ is type-B anchors, and $V_{\text {nonEBF }}$ is the supporting volume for depthaverage conductivity $\mathbf{K}_{A}$ along each of the nonflowmeter wells.

[66] Following Rubin [2003], we can convert $\mathbf{K}_{A}$ to effective conductivity $\overline{\mathbf{9}}=\ln \mathbf{K}_{G}$ along the well using

$$
\overline{\mathbf{\vartheta}}=\ln \mathbf{K}_{A}-\frac{\eta_{\text {local }}^{2}}{2}
$$

where $\eta_{\text {local }}^{2}$ is a local variance at each nonflowmeter well, which is a conditional variance at the midpoint elevation of each non-EBF well conditioned on $\left\{\boldsymbol{\theta}, \boldsymbol{\vartheta}_{a}\right\}$. With equation (A1), realizations of $\left\{\boldsymbol{\theta}, \boldsymbol{\vartheta}_{a}, \mathbf{K}_{A}\right\}$ can be converted to realizations of $\left\{\boldsymbol{\theta}, \boldsymbol{\vartheta}_{a}, \overline{\boldsymbol{9}}\right\}$.

[67] To connect data with different support volumes, a point $\mathbf{x}_{i}$ or a finite volume $V_{i}$, we define three correlation matrices according to the work by Behrens et al. [1998].

[68] 1. Point-to-point correlation:

$$
\mathbf{R}\left(\mathbf{x}_{i}, \mathbf{x}_{j}\right)=\rho\left(\mathbf{x}_{i}, \mathbf{x}_{j}\right),
$$

where $\mathbf{x}_{i}$ and $\mathbf{x}_{j}$ are two point locations and $\rho\left(\mathbf{x}_{i}, \mathbf{x}_{j}\right)$ is the correlation coefficient between these two points.

[69] 2. Point-to-block correlation:

$$
\overline{\mathbf{R}}\left(\mathbf{x}_{i}, V_{j}\right)=\frac{1}{V_{j}} \int_{\mathbf{x}^{\prime} \in \mathbf{x}_{j}} \mathbf{R}\left(\mathbf{x}_{i}, \mathbf{x}^{\prime}\right) d \mathbf{x}^{\prime} .
$$

[70] This represents the correlation between the point $\mathbf{x}_{\mathrm{i}}$ and the volume $V_{j}$. The point-to-point correlation is averaged over the volume $V_{j}$.

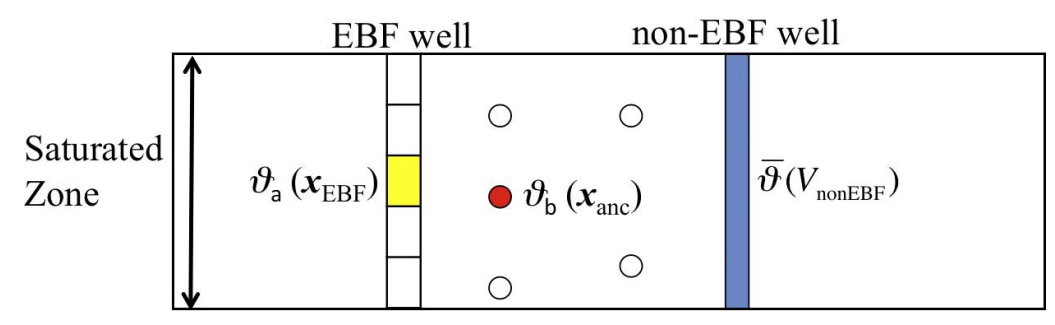

Figure A1. Vertical cross section of the domain including conditional information (EBF refers to electromagnetic borehole flowmeter). 
[71] 3. Block-to-block correlation:

$$
\overline{\mathbf{R}}\left(V_{i}, V_{j}\right)=\frac{1}{V_{i} V_{j}} \int_{\mathbf{x} \in V_{i}} \int_{\mathbf{x}^{\prime} \in V_{j}} \mathbf{R}\left(\mathbf{x}, \mathbf{x}^{\prime}\right) d \mathbf{x} d \mathbf{x}^{\prime}
$$

[72] This represents the correlation between one volume $V_{i}$ and another volume $V_{j}$. The point-to-point correlation is averaged over the two volumes $V_{i}$ and $V_{j}$.

[73] Using the point-to-block and block-to-block correlations, we can include $\overline{\boldsymbol{9}}$ as another set of the conditioning values in the field generation.

[74] Acknowledgments. This work is supported by the U.S. Department of Energy under contract DE-AC05-76RL01830. PFLOTRAN is developed under the DOE Scientific Discovery through Advanced Computing (SciDAC-2) program. The high-performance computation was performed on the Franklin Supercomputer at NERSC, supported by the DOE Office of Science under contract DE-AC02-05CH11231. The unconditional field generation code was provided by Wolfgang Nowak.

\section{References}

Alcolea, A., J. Carrera, and A. Medina (2006), Pilot points method incorporating prior information for solving the groundwater flow inverse problem, Adv. Water Res., 29(11), 1678-1689, doi:10.1016/j.advwatres.2005. 12.009 .

Andrieu, C., N. de Freitas, A. Doucet, and M. I. Jordan (2003), An introduction to MCMC for machine learning, Mach. Learn., 50, 5-43.

Behrens, R. A., M. K. MacLeod, T. T. Tran, and A. O. Alimi (1998), Incorporating seismic attribute maps in 3D reservoir models, SPE Reservoir Eval. Eng., 1(2), 122-126, doi:10.2118/36499-PA.

Bellin, A., and Y. Rubin (2004), On the use of peak concentration arrival times for the inference of hydrogeologic parameters, Water Resour. Res. 40, W07401, doi:10.1029/2003WR002179.

Beven, K. J. (2007), Towards integrated environmental models of everywhere: Uncertainty, data and modelling as a learning process, Hydrol. Earth Syst. Sci., 11(1), 460-467, doi:10.1016/j.jhydrol.2007.02.023.

Beven, K. J., and A. M. Binley (1992), The future of distributed models: Model calibration and uncertainty prediction, Hydrol. Processes, 6, 279298.

Bjornstad, B. N., J. A. Horner, V. R. Vermeul, D. C. Lanigan, and P. D. Thorne (2009), Borehole completion and conceptual hydrolgeologic model for the IFRC well field, 300 Area, Hanford Site, Rep. PNNL18340, Pacific Northwest National Laboratory, Richland, Wash.

Box, G. E. P., and D. R. Cox (1964), An analysis of transformations, J. R. Stat. Soc., Ser. B, 26(2), 211-252.

Burdine, N. (1953), Relative permeability calculations from pore-size distribution data, Trans. Am. Inst. Min. Metall. Eng., 198, 71-78.

Capilla, J. E., J. J. Gómez-Hernández, and A. Sahuquillo (1997), Stochastic simulation of transmissivity fields conditional to both transmissivity and piezometric data, 2. Demonstration on a synthetic aquifer, J. Hydrol., 203, 175-188, doi:10.1016/S0022-1694(97)00097-8.

Cirpka, O. A., and S. Attinger (2003), Effective dispersion in heterogeneous media under random transient flow conditions, Water Resour. Res., 39(9), 1257, doi:10.1029/2002WR001931

Cirpka, O. A., and P. K. Kitanidis (2000a), Sensitivity of temporal moments calculated by the adjoint-state method, and joint inversing of head and tracer data, Adv. Water Resour., 24, 89-103, doi:10.1016/S0309-1708(00)00007-5.

Cirpka, O. A., and P. K. Kitanidis (2000b), Characterization of mixing and dilution in heterogeneous aquifers by means of local temporal moments, Water Resour. Res., 36(5), 1221-1236, doi:10.1029/1999WR900354.

Dagan, G. (1985), Stochastic modeling of groundwater-flow by unconditional and conditional probabilities: The inverse problem, Water Resour. Res., 21(1), 65-72.

de Barros, F. P. J., and Y. Rubin (2011), Modelling of block-scale macrodispersion as a random function, J. Fluid. Mech., 676, 514-545, doi:10. 1017/jfm.2011.65

de Barros, F. P. J., Y. Rubin, and R. M. Maxwell (2009), The concept of comparative information yield curves and its application to risk-based site characterization, Water Resour. Res., 45, W06401, doi:10.1029/ 2008WR007324.
Dentz, M., and J. Carrera (2005), Effective solute transport in temporally fluctuating flow through heterogeneous media, Water Resour. Res., 41(8), W08414, doi:10.1029/2004WR003571.

Diggle, P. J., and P. J. Ribeiro (2006), Model-Based Geostatistics, 230 pp., Springer, N. Y.

Evensen, G. (1994), Sequential data assimilation with a nonlinear quasigeostrophic model using Monte Carlo methods to forecast error statistics, J. Geophys. Res., 99(C5), 10143-10162.

Evensen, G. (2003), The ensemble Kalman filter: Theoretical formulation and practical implementation, Ocean Dynamics, 53(4), 343-367, doi:10.1007/s10236-003-0036-9.

Ezzedine, S., and Y. Rubin (1996), A geostatistical approach to the conditional estimation of spatially distributed solute concentration and notes on the use of tracer data in the inverse problem, Water Resour. Res., 32, 853-861, doi:10.1029/95WR02285.

Fienen, M. N., T. Clemo, and P. K. Kitanidis (2008), An interactive Bayesian geostatistical inverse protocol for hydraulic tomography, Water Resour. Res., 44, W00B01, doi:10.1029/2007WR006730.

Fienen, M. N., R. J. Hunt, D. P. Krabbenhoft, and T. Clemo (2009), Obtaining parsimonious hydraulic conductivity fields using head and transport observations: A Bayesian geostatistical parameter estimation approach, Water Resour. Res., 45, W08405, doi:10.1029/2008WR007431.

$\mathrm{Fu}$, J., and J. J. Gómez-Hernández (2009), Uncertainty assessment and data worth in groundwater flow and mass transport modeling using a blocking Markov chain Monte Carlo method, J. Hydrol., 364, 328-341, doi:10.1016/j.jhydrol.2008.11.014.

Gómez-Hernández, J. J., A. Sahuquillo, and J. E. Capilla (1997), Stochastic simulation of transmissivity fields conditional to both transmissivity and piezometric data, 1. Theory, J. Hydrol., 203, 162-174, doi:10.1016/ S0022-1694(97)00098-X.

Goode, D. J., and L. F. Konikow (1990), Apparent dispersion in transient groundwater flow, Water Resour. Res., 26(10), 2339-2351, doi:10.1029/ WR026i010p02339.

Hammond, G. E., and P. C. Lichtner (2010), Field-scale model for the natural attenuation of uranium at the Hanford 300 Area using highperformance computing, Water Resour. Res., 46, W09527, doi:10.1029/ 2009WR008819.

Harvey, C. F., and S. M. Gorelick (1995a), Mapping hydraulic conductivity: Sequential conditioning with measurements of solute arrival time, hydraulic-head, and local conductivity, Water Resour. Res., 31, 16151626, doi:10.1029/95WR00547.

Harvey, C. F., and S. M. Gorelick (1995b), Temporal moment-generating equations: Modeling transport and mass transfer in heterogeneous aquifers, Water Resour. Res., 31(8), 1895-1911, doi:10.1029/95WR01231.

Hayfield, T., and J. S. Racine (2008), Nonparametric econometrics: The NP package, J. Stat. Software, 27(5), 1-32.

Hendricks Franssen, H.-J. H., J. J. Gómez-Hernández, and A. Sahuquillo (2003), Coupled inverse modeling of groundwater flow and mass transport and the worth of concentration data, J. Hydrol., 281, 281-295, doi: 10.1016/S0022-1694(03)00191-4.

Hendricks Franssen, H. J., A. Alcolea, M. Riva, M. Bakr, N. van de Wiel, F. Stauffer, and A. Guadagnini (2009), A comparison of seven methods for the inverse modelling of groundwater flow. Application to the characterisation of well catchments. Adv. Water Resour., 32(6), 851-872, doi:10.1016/j.advwatres.2009.02.011.

Hoeksema, R. J., and P. K. Kitanidis (1984), An application of the geostatistical approach to the inverse problem in two-dimensional groundwater modeling, Water Resour. Res., 20, 1003-1020.

Kitanidis, P. K. (1995), Quasi-linear geostatistical theory for inversing, Water Resour. Res., 31(10), 2411-2419, doi:10.1029/95WR01945.

Kitanidis, P. K. (1998), How observations and structure affect the geostatistical solution to the steady- state inverse problem, Ground Water, 36(5), 754-763, doi:10.1111/j.1745-6584.1998.tb02192.x.

Kollat, J. B., P. M. Reed, and D. M. Rizzo (2008), Addressing model bias and uncertainty in three dimensional groundwater transport forecasts for a physical aquifer experiment, Geophys. Res. Lett., 35, L17402, doi:10.1029/ 2008 GL035021.

Kollat, J. B., P. M. Reed, and R. M. Maxwell (2011), Many-objective groundwater monitoring network design using bias- aware ensemble Kalman filtering, evolutionary optimization, and visual analytics, Water Resour. Res., 47, W02529, doi:10.1029/2010WR009194.

LaVenue, A. M., B. S. RamaRao, G. de Marsily, and M. G. Marietta (1995), Pilot point methodology for automated calibration of an ensemble of conditionally simulated transmissivity fields, 2 . Application, Water Resour. Res., 31(3), 495-516, doi:10.1029/94WR02259. 
Lavenue, M., and G. de Marsily (2001), Three-dimensional interference test interpretation in a fractured aquifer using the Pilot Point Inverse Method, Water Resour. Res., 37(11), 2659-2675, doi:10.1029/2000 WR000289.

Li, W., A. Englert, O. A. Cirpka, and H. Vereecken (2008), Threedimensional geostatistical inversion of flowmeter and pumping test data, Ground Water, 46(2), 193-201, doi:10.1111/j. 1745-6584.2007. 00419.x.

Mantovan, P., and E. Todini (2006), Hydrological forecasting uncertainty assessment: Incoherence of the GLUE methodology, J. Hydrol., 330(1-2), 368-381, doi:10.1016/j.jhydrol.2006.04.046.

McKenna, S. A., J. Doherty, and D. B. Hart (2003), Non-uniqueness of inverse transmissivity field calibration and predictive transport modeling, J. Hydrol., 281(4), 265-280, doi:10.1016/S0022-1694(03)00194-X.

McLaughlin, D. and L. R. Townley (1996), A reassessment of the groundwater inverse problem, Water Resour. Res., 32(5), 1131-1161, doi:10. 1029/96WR00160

Medina, A., and J. Carrera (2003), Geostatistical inversion of coupled problems: dealing with computational burden and different types of data, $J$. Hydrol., 281, 251-264, doi:10.1016/S0022-1694(03)00190-2.

Molz, F. J., G. K. Boman, S. C. Young, and W. R. Waldrop (1994), Borehole flowmeters: Field application and data analysis, J. Hydrol., 163 , 347-371, doi:10.1016/0022-1694(94)90148-1.

Moradkhani, H., K. L. Hsu, H. Gupta, and S. Sorooshian (2005), Uncertainty assessment of hydrologic model states and parameters: Sequential data assimilation using the particle filter, Water Resour. Res., 41(5), W05012, doi:10.1029/2004WR003604.

Murakami, H., X. Chen, M. S. Hahn, Y. Liu, M. L. Rockhold, V. R. Vermeul, J. Zachara, and Y. Rubin (2010), Bayesian approach for three-dimensional aquifer characterization at the Hanford 300 Area, Hydrol. Earth Syst. Sci., 14, 1989-2001, doi:10.5194/hess-14-19892010.

Newcomer, D. R., B. N. Bjornstad, and V. R. Vermeul (2010), Vertical wellbore flow monitoring for assessing spatial and temporal flow relationships with a dynamic river boundary, Ground Water Monit. Rem., 30(4), 123-135, doi:10.1111/j.1745-6592.2010.01304.x.

Nowak, W., and O. A. Cirpka (2006), Geostatistical inference of conductivity and dispersion coefficients from hydraulic heads and tracer data, Water Resour. Res., 42, W08416, doi:10.1029/2005WR004832.

Nowak, W., S. Tenkleve, and O. A. Cirpka (2003), Efficient computation of linearized cross-covariance and auto-covariance matrices of interdependent quantities, Math. Geol., 35(1), 53-66, doi:10.1023/A:1022 365112368 .

Nowak, W., F. P. J. de Barros, and Y. Rubin (2010), Bayesian geostatistical design: Task-driven optimal site investigation when the geostatistical model is uncertain, Water Resour. Res., 46, W03535, doi:10.1029/ 2009WR008312.

Peterson, R. E., M. L. Rockhold, R. J. Serne, P. D. Thorne, and M. D. Williams (2008), Uranium contamination in the subsurface beneath the 300 Area, Hanford Site, Washington, Rep. PNNL-17034, Pacific Northwest National Laboratory, Richland, Wash.

Pollock, D., and O. A. Cirpka (2010), Fully coupled hydrogeophysical inversion of synthetic salt tracer experiments, Water Resour. Res., 46, W07501, doi:10.1029/2009WR008575.

RamaRao, B. S., A. M. LaVenue, G. de Marsily, and M. G. Marietta (1995), Pilot point methodology for automated calibration of an ensemble of conditionally simulated transmissivity fields, 1 . Theory and computational experiments, Water Resour. Res., 31(3), 475-493, doi:10.1029/ 94WR02258.

Rockhold, M. L., S. R. Wachler, D. L. Saunders, R. E. Clayton, and C. E. Strickland (2009), Soil water balance and recharge monitoring at the Hanford Site-FY09 Status Report, PNNL-18807, Pacific Northwest National Laboratory, Richland, Wash.

Rubin, Y. (2003), Applied Stochastic Hydrogeology, 416 pp., Oxford Univ. Press, Cambridge, U. K.
Rubin, Y., and G. Dagan (1987), Stochastic identification of transmissivity and effective recharge in steady groundwater flow: 1 . Theory, Water Resour. Res., 23(7), 1185-1192, doi:10.1029/WR023i007p01185.

Rubin, Y., A. Sun, R. Maxwell, and A. Bellin (1999), The concept of block effective macrodispersion and a unified approach for grid-scale- and plume-scale-dependent transport, J. Fluid Mech., 395, 161-180.

Rubin, Y., A. Bellin, and A. Lawrence (2003), On the use of block-effective macrodispersion for numerical simulation of transport in heterogeneous formations, Water Resour. Res., 39(9), 1242, doi:10.1029/2002WR001727.

Rubin, Y., X. Chen, H. Murakami, and M. Hahn (2010), A Bayesian approach for data assimilation and conditional simulation of spatial random fields, Water Resour. Res., 46, W10523, doi:10.1029/2009WR008799.

Sahuquillo, A., J. E. Capilla, J. J. Gómez-Hernández, and J. Andreu (1992), Conditional simulation of transmissivity fields honoring piezometric data, in Hydraulic Engineering Software IV, Fluid Flow Modeling, 2, edited by W. R. Blain and E. Cabrera, pp. 201-214, Elsevier, N. Y.

Scheibe, T. D., Y.-J. Chien, and J. Radtke (2001), Use of quantitative models to design microbial transport experiments in a sandy aquifer, Ground Water, 39(2), 210-222.

Scott, D. W., and S. R. Sain (2004), Multi-dimensional density estimation, in Handbook of Statistics, vol 23: Data Mining and Computational Statistics, edited by C. R. Rao and E. J. Wegman, pp. 229-263, Elsevier, Amsterdam, Netherlands.

Shlomi, S., and A. M. Michalak (2007), A geostatistical framework for incorporating transport information in estimating the distribution of a groundwater contaminant plume, Water Resour. Res., 43, W03412, doi:10.1029/ 2006WR005121.

Sudicky, E. A., W. A. Illman, I. K. Goltz, J. J. Adams, and R. G. McLaren (2010), Heterogeneity in hydraulic conductivity and its role on the macroscale transport of a solute plume: From measurements to a practical application of stochastic flow and transport theory, Water Resour. Res., 46(1), W01508, doi:10.1029/2008WR007558.

van Genuchten, M. T. (1980), A closed-form equation for predicting the hydraulic conductivity of unsaturated soils, Soil Sci. Soc. Am. J., 44, 892-898. Vermuel, V. R., J. P. Mckinley, D. R. Newcomer, R. D. Mackley, and J. M. Zachara (2010), River-induced flow dynamics in long-screen wells and impact on aqueous samples, Ground Water, 49(4), 515-524, doi:10. 1111/j.1745-6584.2010.00769.x.

Vrugt, J. A., P. H. Stauffer, T. Wöhling, B. A. Robinson, and V. V. Vesselinov (2008), Inverse modeling of subsurface flow and transport properties: A review with new developments, Vadose Zone J., 7(2), 843-864, doi:10.2136/vzj2007.0078.

Williams, M. D., M. L. Rockhold, P. D. Thorne, and Y. Chen (2008), Three-dimensional groundwater models of the 300 Area at the Hanford Site, Washington State, Rep. PNNL-17708, Pacific Northwest National Laboratory, Richland, Wash.

Wilson, A., and Y. Rubin (2002), Characterization of aquifer heterogeneity using indicator variables for solute concentrations, Water Resour. Res. 38(12), 1283, doi:10.1029/2000WR000116.

Woodbury, A. D., and Y. Rubin (2000), A full-Bayesian approach to parameter inference from tracer travel time moments and investigation of scale effects at the Cape Cod Experimental Site, Water Resour. Res., 36(1), 159-171, doi:10.1029/1999WR900273.

Zachara, J. M. (2010), Multi-scale mass transfer processes controlling natural attenuation and engineered remediation: An IFRC focused on Hanford's 300 Area Uranium Plume, Rep. PNNL-19209, Pacific Northwest National Laboratory, Richland, Wash.

Zimmerman, D. A., et al. (1998), A comparison of seven geostatistically based inverse approaches to estimate transmissivities for modeling advective transport by groundwater flow, Water Resour. Res., 34(6), 13731413, doi: 10.1029/98WR00003.

Zinn, B., and C. F. Harvey (2003), When good statistical models of aquifer heterogeneity go bad: A comparison of flow, dispersion, and mass transfer in connected and multivariate Gaussian hydraulic conductivity fields, Water Resour. Res., 39(3), 1051, doi:10.1029/2001WR001146. 